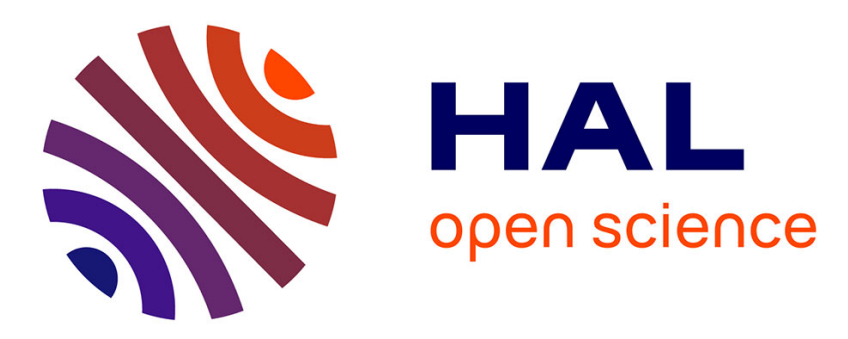

\title{
Full-field polycrystal plasticity simulations of neutron-irradiated austenitic stainless steel: A comparison between FE and FFT-based approaches
}

S. El Shawish, P.-G. Vincent, H. Moulinec, L. Cizelj, L. Gélébart

\section{- To cite this version:}

S. El Shawish, P.-G. Vincent, H. Moulinec, L. Cizelj, L. Gélébart. Full-field polycrystal plasticity simulations of neutron-irradiated austenitic stainless steel: A comparison between FE and FFT-based approaches. Journal of Nuclear Materials, 2020, 529, pp.151927. 10.1016/j.jnucmat.2019.151927 . hal-02968799

\section{HAL Id: hal-02968799 \\ https://hal.science/hal-02968799}

Submitted on 7 Jan 2021

HAL is a multi-disciplinary open access archive for the deposit and dissemination of scientific research documents, whether they are published or not. The documents may come from teaching and research institutions in France or abroad, or from public or private research centers.
L'archive ouverte pluridisciplinaire HAL, est destinée au dépôt et à la diffusion de documents scientifiques de niveau recherche, publiés ou non, émanant des établissements d'enseignement et de recherche français ou étrangers, des laboratoires publics ou privés.

\section{(ㅇ)(1) $\$$}

Distributed under a Creative Commons Attribution - NonCommercial - NoDerivatives| 4.0 


\title{
Full-field polycrystal plasticity simulations of neutron-irradiated austenitic stainless steel: a comparison between FE and FFT-based approaches
}

\author{
S. El Shawish ${ }^{\mathrm{a}, *}$, P.-G. Vincent ${ }^{\mathrm{b}}$, H. Moulinec ${ }^{\mathrm{c}}$, L. Cizelj $^{\mathrm{a}}$, L. Gélébart ${ }^{\mathrm{d}}$ \\ ${ }^{a}$ Jožef Stefan Institute, SI-1000, Ljubljana, Slovenia \\ ${ }^{b}$ Institut de Radioprotection et de Sûreté Nucléaire, B.P. 3, 13115 Saint-Paul-lez-Durance Cedex, France \\ ${ }^{c}$ Aix-Marseille Univ, CNRS, Centrale Marseille, LMA, 4 Impasse Nikola Tesla, CS 40006, 13453 Marseille Cedex 13, France \\ ${ }^{d}$ DEN-Service de Recherches Métallurgiques Appliquées, CEA, Université Paris-Saclay, F-91191, Gif-sur-Yvette, France
}

\begin{abstract}
We compare two full-field approaches - a crystal plasticity finite element method (CP-FEM) and crystal plasticity fast Fourier transform-based (CP-FFT) method - for a specific crystal plasticity law introduced for neutron-irradiated austenitic stainless steel SA304L currently used in nuclear reactor vessel internals. This particular law is employed to identify and quantify possible advantages and drawbacks of the two approaches when used in the large-scale simulations to predict the effect of irradiation damage (e.g., crack initiation) in stainless steel microstructures. A comparison is performed in a polycrystalline context for different periodic Voronoi microstructures deformed under tension. Special emphasis is put on studying the performance of the two approaches in terms of mesh convergence analysis using aggregate models with different spatial discretizations. In the CP-FEM approach, the performance of linear as well as quadratic tetrahedral meshes is investigated. A similar performance between the CP-FEM and CP-FFT methods is demonstrated in a smaller 2-grain aggregate. However, a slower mesh convergence is observed for the CPFEM method when comparing tensile responses of a larger 100-grain polycrystal. A drop in the convergence rate is much more pronounced on linear than on quadratic tetrahedral meshes. As a consequence, largest (average) grain boundary stresses are shown to be overestimated with the linear mesh CP-FEM approach, thus raising a concern of possible over-conservatism employed in the CP-FEM prediction of crack initiation in irradiated stainless steels. On the contrary, the mesh convergence of the CP-FFT approach is found to be practically independent of the applied macroscopic strain and also aggregate size. Therefore, for such steels, the CP-FFT approach seems to be better justified.
\end{abstract}

Keywords: Crystal plasticity, Irradiation hardening, Finite element simulations, Fast Fourier Transforms simulations, Austenitic stainless steel

\footnotetext{
* Corresponding authors:

Email address: samir.elshawish@ijs.si (S. El Shawish)
} 


\section{Introduction}

In a pressurized water reactor (a widespread type of nuclear reactor), the reactor vessel internals are structural components which have many functions. They are used to support the core, control rod assemblies, core support structure, and reactor pressure vessel surveillance capsules. They are also used to direct the flow of the reactor coolant and to provide shielding for the reactor pressure vessel [1]. In western type pressurized water reactors, the internals are also associated with many safety functions such as to support the core, maintain the reactivity control, assure the core cooling, and assure the instrumentation availability. The internals are mainly made of austenitic stainless steels because of their corrosion resistance, toughness, ductility, strength and fatigue characteristics in pressurized water reactor environment (see [1] for a more complete description of the reactor vessel internals). Their operating conditions depend on the type of reactor and also on their location inside the core. For example, [1] indicates some estimates of the temperature of operation of the different parts of the internals in French 1300 MWe nuclear power plants between $286^{\circ} \mathrm{C}$ and $370^{\circ} \mathrm{C}$. Moreover, the internals are exposed to neutron irradiation which may induce large changes in microstructure (radiation hardening) and microchemistry (radiation-induced segregation) of the austenitic stainless steels and degrade their fracture properties [2]. Irradiation-assisted stress corrosion cracking (IASCC) is another degradation process [3]. It corresponds to an increased susceptibility to stress corrosion cracking in irradiated materials. This is a complex phenomenon that involves simultaneous action of neutron irradiation, applied stress, and corrosive environment [4]. As pointed out in [4], irradiation hardening and embrittlement are key irradiation effects that have significant impact on IASCC behavior of austenitic stainless steels.

Specific crystal plasticity laws have been recently developed to model irradiation hardening in austenitic stainless steels at the grain scale $[5,6,7,8]$. Full-field simulations on polycrystalline aggregates using such crystal plasticity laws within the finite element framework have been performed to estimate the overall elasto-viscoplastic response of these steels. Also, such crystal plasticity laws have been used to estimate the intergranular (normal) stress distributions as normal stresses at grain boundaries are believed to be a key parameter in predicting IASCC initiation $[9,10]$.

The present study compares two approaches, Finite Element Method (FEM) simulations performed with Abaqus software [11] and Fast Fourier Transforms (FFT) based simulations performed with CraFT software as presented in [12], applied to irradiated austenitic stainless steel SA304L currently used for reactor vessel internals. A general comparison between the FEM crystal plasticity (CP-FEM) and FFT crystal plasticity (CP-FFT) methods may be found, e.g., in [13]. In short, a variational solution is achieved in the CP-FEM for the equilibrium of the forces and the compatibility of the displacements using the principle of virtual work for a volume that is discretized into finite elements. In the CP-FFT, a FFT-based algorithm is applied in conjunction with Green's function method to solve the governing equations for heterogeneous media. This 
approximation solves the equilibrium equations under the constraint of strain compatibility for materials with periodic microstructure, which can be generated by periodic repetition of a unit cell. Contrary to the CP-FEM, no meshing is needed in the CP-FFT approach. Instead, the model geometry (e.g., the microstructure) is described by an image composed of voxels. In general, for the same spatial resolution, the CP-FFT approach is a very efficient alternative compared with the CP-FEM with periodic boundary conditions. Better numerical performance of the CP-FFT is related to the repetitive use of the efficient FFT algorithm, avoiding the time-consuming resolution of linear systems needed under the CP-FEM.

The main objective of this study is to compare the accuracy and performance of the CP-FEM and CP-FFT methods when applied to irradiated austenitic stainless steel SA304L currently used for reactor vessel internals, to check if they provide similar results (in terms of stresses), and to evaluate their potential discrepancies. From a practical point of view, such comparison is also important to identify and quantify possible advantages and drawbacks of the methods when used in large-scale analyses to predict the effects of irradiation damage, i.e., IASCC initiation, in a representative volume element of the microstructure. In particular, it would be interesting to check if the CP-FFT model discretization into regular voxel grid is suitable to provide also accurate stresses on the approximately resolved grain boundaries.

While there have been previous comparisons performed between the CP-FEM and CP-FFT simulations [13], [14], [15], [16], they all have made use of more standard crystal plasticity constitutive equations. Here, the study employs a very specific crystal plasticity law devoted to SA304L irradiated stainless steel: the hardening law accounts for the mobile dislocation density evolution and also various defects created by irradiation. As a result, such crystal plasticity law involves a large number of internal variables, which implies high computational costs when employed in a polycrystalline context.

The paper is organized as follows. The methods and models are described in Sec. 2, results are presented and discussed in Sec. 3 and conclusions are given in Sec. 4.

\section{Methods and models}

\subsection{Constitutive crystal plasticity law}

To perform a meaningful comparison between the CP-FEM and CP-FFT methods, the same constitutive relationship was implemented within both models. A micromechanical crystal plasticity model recently developed by french Commissariat à l'Energie Atomique (CEA) [6, 7, 9] was used here to study the tensile response of the austenitic stainless steel SA304L irradiated to $0.8 \mathrm{dpa}$ (displacements per atom). The model is able to capture the irradiation-induced hardening followed by softening during plastic deformation. For completeness, the constitutive model is briefly described below.

The material model for irradiated SA304L consists of anisotropic elasticity and crystal plasticity. The anisotropic elasticity is governed by the stiffness tensor with three independent parameters, $C_{11}, C_{12}, C_{44}$, 
which are well known for SA304L (see Tab. 1, Voigt notation). The plastic model takes into account the evolution of dislocation densities and irradiation defects - Frank loops [17].

The shear flow adopted here for irradiated conditions is of visco-plastic type and represents isotropic hardening,

$$
\dot{\gamma}^{\alpha}=\left[\frac{\left|\tau^{\alpha}\right|-\tau_{c}^{\alpha}}{K_{0}}\right]^{n} \operatorname{sign}\left(\tau^{\alpha}\right), \quad \text { with } \quad[x]= \begin{cases}x & ; x>0 \\ 0 & ; x \leq 0\end{cases}
$$

where $\gamma^{\alpha}$ is shear strain in slip system $\alpha\left(\alpha=1 \ldots 12\right.$ for face-centered-cubic lattice) and $\tau^{\alpha}$ and $\tau_{c}^{\alpha}$ are respectively the resolved shear stress and critical resolved shear stress. Parameters $K_{0}$ and $n$ regulate the viscosity of the shear flow.

The critical resolved shear stress is additively decomposed into components that contribute to hardening,

$$
\tau_{c}^{\alpha}=\tau_{0}+\tau_{a} \mathrm{e}^{-\frac{\left|\gamma^{\alpha}\right|}{\gamma_{0}}}+\mu \sqrt{\sum_{\beta=1}^{12} a^{\alpha \beta} r_{D}^{\beta}}+\mu \alpha_{L} \sqrt{\sum_{p=1}^{4} r_{L}^{p}} .
$$

Here, $r_{D}^{\alpha}$ is the normalized dislocation density in slip system $\alpha, \tau_{0}$ is the lattice friction stress that remains constant for a given temperature, while $\mu$ and $a^{\alpha \beta}$ are respectively the macroscopic shear modulus and a $12 \times 12$ hardening matrix (with 6 independent parameters) accounting for interactions between dislocations. In the irradiated material, an additional hardening is expected due to presence of Frank loops. Here, $r_{L}^{p}$ is a normalized Frank loop density in slip plane $p$ and $\alpha_{L}$ is an effective force on the Frank loop obstacle. To account for a dislocation unlock mechanism [6,7], an additional phenomenological term has been proposed along with a dose dependent shear stress $\tau_{a}$ required to unlock the dislocations and coefficient $\gamma_{0}$ to adjust the speed of the avalanche after unlocking the dislocations. Note, however, that this avalanche term is assumed negligible in SA304L at 0.8 dpa (thus $\tau_{a}=0$ ) [9].

The evolution of dislocation density is modeled with a multiplication and an annihilation term,

$$
\dot{r}_{D}^{\alpha}=\left(\frac{1}{\kappa} \sqrt{\sum_{\beta=1}^{12} b^{\alpha \beta} r_{D}^{\beta}}+\frac{1}{\kappa} \sqrt{K_{d l} \sum_{p=1}^{4} r_{L}^{p}}-G_{c} r_{D}^{\alpha}\right)\left|\dot{\gamma}^{\alpha}\right|
$$

where $b^{\alpha \beta}$ is a matrix of interactions between dislocations, being of the same shape as $a^{\alpha \beta}$. Parameter $\kappa$ is proportional to the number of obstacles crossed by a dislocation before being immobilized and $G_{c}$ is a proportional factor that depends on the annihilation mechanism of dislocation dipoles. The irradiation effects are modeled by adding a term to the multiplication part, with $K_{d l}$ being a coefficient of effective interaction between dislocations and Frank loops.

The evolution of Frank loop density is modeled by [18]

$$
\dot{r}_{L}^{p}=-A_{L}\left(r_{L}^{p}-r_{L}^{s a t}\right)\left(\sum_{\alpha \in \text { plane } p}^{3} r_{D}^{\alpha}\right)\left(\sum_{\alpha \in \text { plane } p}^{3}\left|\dot{\gamma}^{\alpha}\right|\right)
$$

where $A_{L}$ is the annihilation area of Frank loops and $r_{L}^{s a t}$ is a stabilized value of the normalized defect density which depends on the irradiation dose. Since scanning of Frank loops by mobile dislocations occurs only 


\begin{tabular}{ccccccc}
$C_{11}$ & $C_{12}$ & $C_{44}$ & $\mu$ & $K_{0}$ & $\tau_{0}$ & $\tau_{a}$ \\
\hline $199 \mathrm{GPa}$ & $136 \mathrm{GPa}$ & $105 \mathrm{GPa}$ & $65.615 \mathrm{GPa}$ & $10 \mathrm{MPa} \mathrm{s}{ }^{1 / n}$ & $88 \mathrm{MPa}$ & $0 \mathrm{MPa}$
\end{tabular}

\begin{tabular}{cccccccccccccc}
$n$ & $G_{c}$ & $\kappa$ & $a_{1}$ & $a_{2}$ & $a_{3}$ & $a_{4}$ & $a_{5}$ & $a_{6}$ & $b_{i}$ & $r_{D}^{0}$ & $r_{L}^{0}$ & $K_{d l}$ & $\alpha_{L}$ \\
\hline 15 & 10.4 & 42.8 & 0.124 & 0.124 & 0.070 & 0.625 & 0.137 & 0.122 & $1-\delta_{1 i}$ & $4.5410^{-11}$ & $2.2910^{-6}$ & $2.5010^{-7}$ & 0.21
\end{tabular}

Table 1: Model parameters for SA304L stainless steel at $330^{\circ} \mathrm{C}$ and irradiated to 0.8 dpa, taken from [9]. Top row: parameters in absolute units. Bottom row: normalized parameters. Note that $r_{L}^{s a t}=r_{L}^{0}$ is set for 0.8 dpa. Index in $b_{i}$ and $\delta_{1 i}$ runs from 1 to 6 .

${ }^{1}$ Using the small-strain approach may lead to wrong deformations of the model that is not constrained enough. This happens because, in small-strain approach, also rotations contribute to the strain, thus a correct strain state may be achieved by combining wrong stretches with wrong rotations. However, although final deformations of the model might be wrong, stresses and strains remain unaffected. 


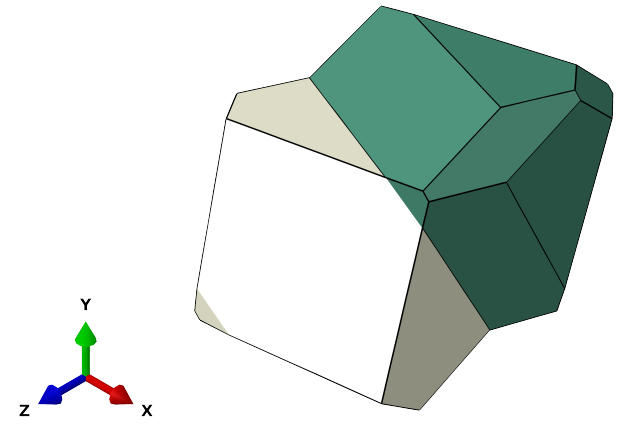

(a) Overall geometry.
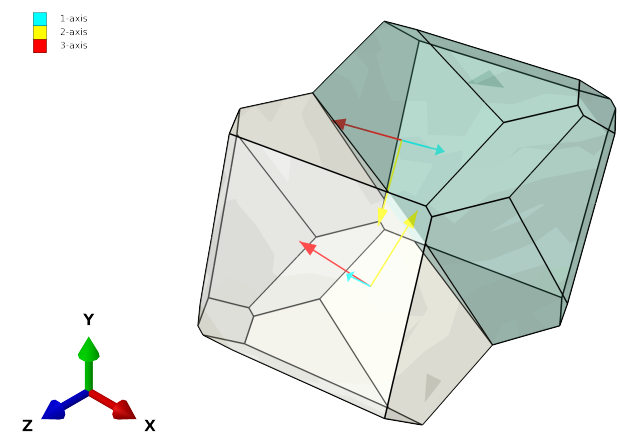

(b) Grain orientations.

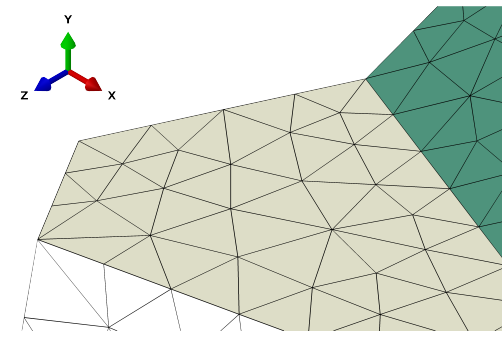

(c) Zoomed: $2.610^{4}$ elements.

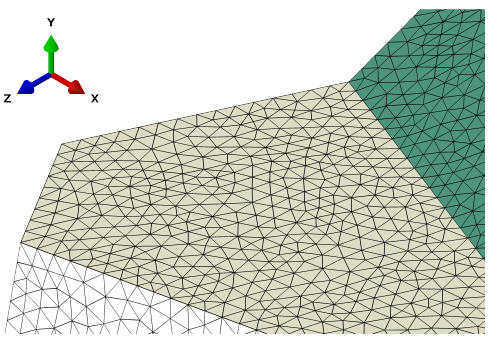

(d) Zoomed: $1.610^{6}$ elements.

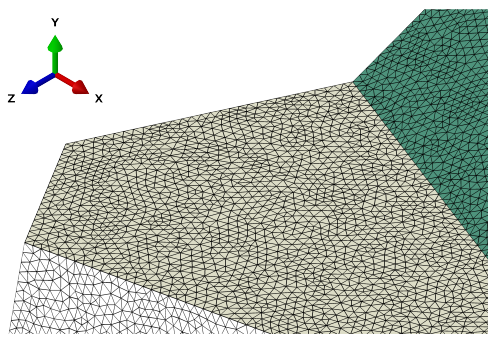

(e) Zoomed: $1.310^{7}$ elements.

Figure 1: Periodic bicrystal aggregate model used in the CP-FEM (and CP-FFT) simulations. Linear (C3D4) and quadratic (C3D10) tetrahedral elements were used in the CP-FEM meshes.

grains was based on a Poisson-Voronoi diagram. Other types of tessellations could be investigated, such as those of Johnson-Mehl-Avrami-Kolmogorov and Laguerre (see [22]). Keeping in mind that the curvy grain boundaries of such tessellations could lead to meshing problems for finite element calculation (non convexity problems), the polycrystals were limited to Voronoi tessellation. The same material properties were assigned to all the grains of the model but with different crystallographic orientations to model either a bicrystal or polycrystal aggregates.

In the CP-FEM models finite element meshes were also generated with Neper [21] using linear tetrahedral elements (C3D4) or quadratic tetrahedral elements (C3D10) in order to preserve the geometry of the grains. ${ }^{2}$ First-order tetrahedral elements are known for their poor performance, especially in situations with high stress or strain gradients; the elements are overly stiff and exhibit rather slow convergence with mesh refinement. Fine meshes are thus often required to obtain results of sufficient accuracy. For this reason, very high mesh densities (with up to 13 million elements, see Fig. 1(e)), limited by our computational resources, were investigated in the CP-FEM simulations. In parallel, similar analyses were performed on second-order meshes, having exactly the same topology of finite elements as the fist-order ones. In this way, the influence

\footnotetext{
${ }^{2}$ We note that quadratic tetrahedral elements (C3D10) are currently not supported in Neper [21] when used in combination with periodic geometries, therefore an in-house script was developed to generate second-order meshes.
} 


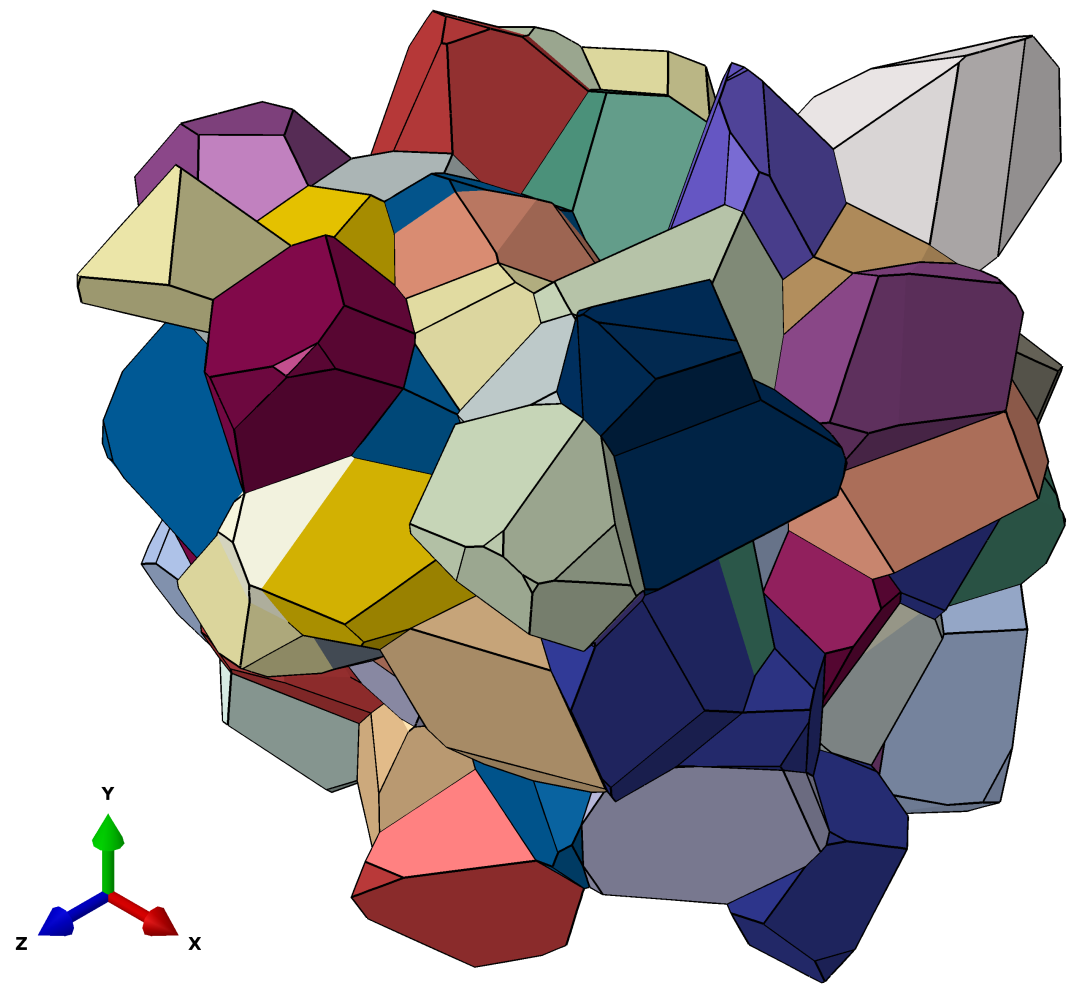

(a) Overall geometry with approximately zero texture.

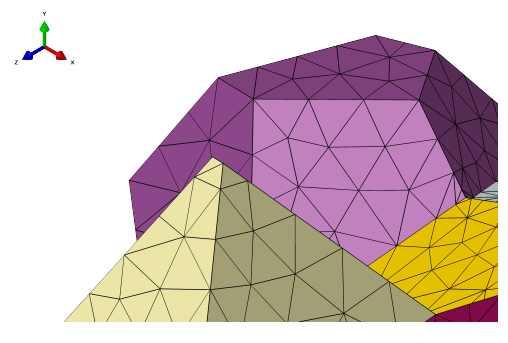

(b) Zoomed: $1.210^{5}$ elements.

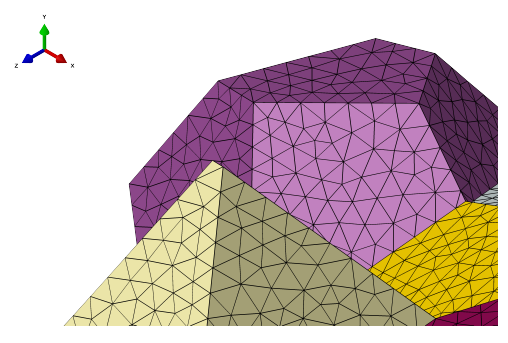

(c) Zoomed: $1.410^{6}$ elements.

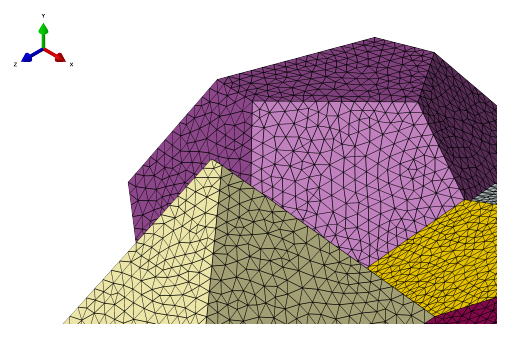

(d) Zoomed: $1.210^{7}$ elements.

Figure 2: Periodic polycrystal aggregate model with 100 grains denoted by different colors used in the CP-FEM (and CP-FFT) simulations. Linear (C3D4) and quadratic (C3D10) tetrahedral elements were used in the CP-FEM meshes.

of element order (linear versus quadratic) on CP-FEM mesh convergence performance could be examined systematically.

The conformal meshing between the neighboring grains allowed us to apply periodic boundary conditions on all of the free faces of the periodic microstructure. Periodic boundary conditions were implemented in Abaqus by introducing three master nodes $M_{j}$, one master node for each space direction $(j=1,2,3)$, and using equation constraints to relate the node displacements on the opposite faces of the aggregate. To simulate the uniaxial tension in the CP-FEM along direction 3 ( $z$ axis), an incremental displacement, $u_{3}\left(M_{3}\right)>0$, was applied to the master node $M_{3}$ which was attached to the two aggregate faces facing the uniaxial direction 3. In this way, the only nonzero macroscopic stress component, $\Sigma_{33}=\left\langle\sigma_{33}\right\rangle>0$, was obtained. Here, the averaging $\langle$.$\rangle was defined over the entire volume of the model.$

In the CP-FFT models the spatial discretization of the volume is based on a regular grid, the basic element 


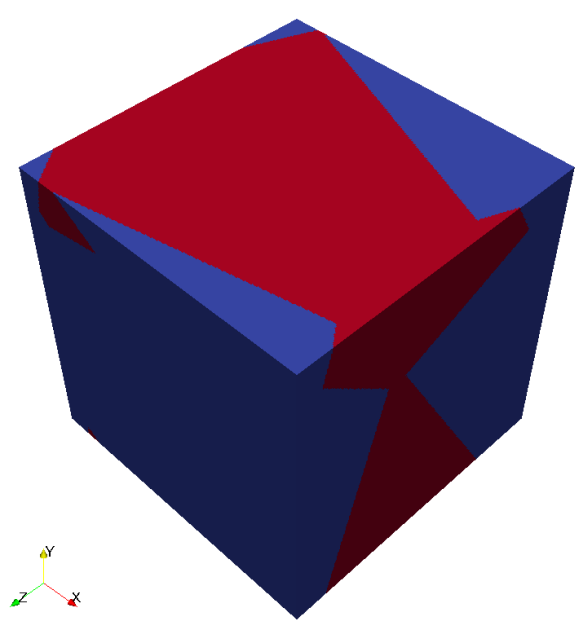

(a) Overall view: $256^{3}$ voxels.

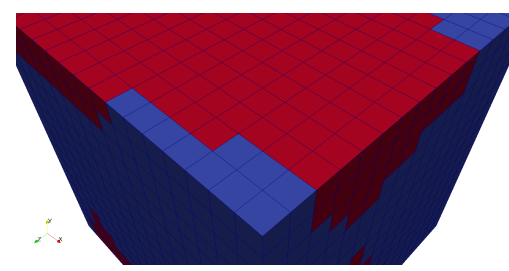

(b) Zoomed: $16^{3}$ voxels

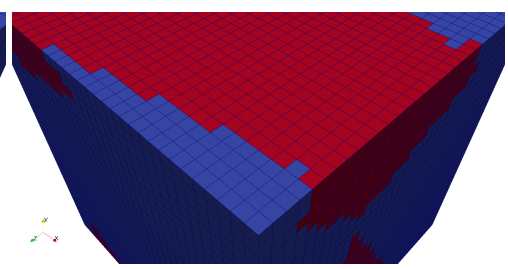

(c) Zoomed: $32^{3}$ voxels.



(d) Zoomed: $64^{3}$ voxels.

(e) Zoomed: $128^{3}$ voxels.

Figure 3: Periodic bicrystal aggregate models used in the CP-FFT simulations (discretization given in voxels). Note that same grain geometry was used as in Fig. 1.

of which is a voxel. For each microstructure, several simulations were performed at different resolutions (i.e. by varying the numbers of voxels in the volume considered). The discretized microstructures are shown in Figs. 3 and 4. Similarly to the CP-FEM, in the CP-FFT simulations the macroscopic stress and strain-rate were defined as volume averages of local stress and strain-rate fields, $\Sigma_{i j}=\left\langle\sigma_{i j}\right\rangle$ and $\dot{E}_{i j}=\left\langle\dot{\epsilon}_{i j}\right\rangle$. From a practical point of view, imposing the direction of the overall stress is often required, rather than the overall strain-rate [23]. A mixed procedure was adopted here [24]: the direction $\boldsymbol{\Sigma}^{o}$ of the overall stress was prescribed together with the strain-rate in this direction. All the computations performed in the present work considered the case of a uniaxial tension in direction 3 ( $z$ axis). The macroscopic stress had only one non-vanishing component $\Sigma_{33}>0$ in the tensile direction and the loading was applied by controlling the strain $E_{33}$. The CP-FFT simulations were performed using small-strain approach in CraFT software, based on the FFT method initially proposed by [25] and [26] to investigate the effective properties of the periodic composites.

The first algorithm developed on that basis is called the basic scheme. In the general algorithm, a convergence test is required to check when the iterating process can be stopped. As in [27], where the CPFFT simulations were recently performed with CraFT on porous viscoplastic crystals, the computations here were carried out using the basic scheme which ensures strain compatibility, together with the convergence criteria on the local equilibrium condition and the prescribed direction of the macroscopic stress. At each iterate $k$, the errors serving to check the convergence are defined as:

$$
\operatorname{err}_{1}(k)=\frac{\left\langle\left\|\operatorname{div} \boldsymbol{\sigma}^{(k)}\right\|^{2}\right\rangle^{1 / 2}}{\left\|\left\langle\boldsymbol{\sigma}^{(k)}\right\rangle\right\|}
$$




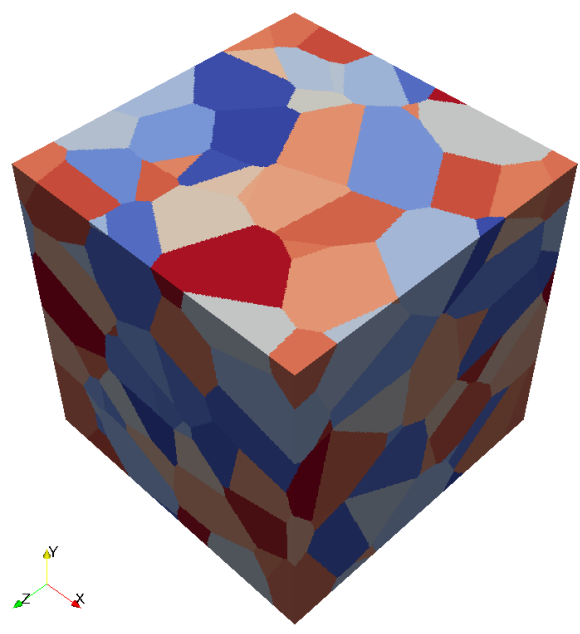

(a) Overall view: $256^{3}$ voxels.

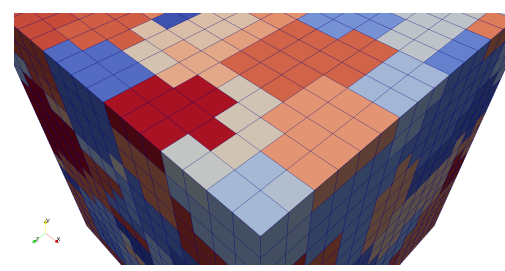

(b) Zoomed: $16^{3}$ voxels

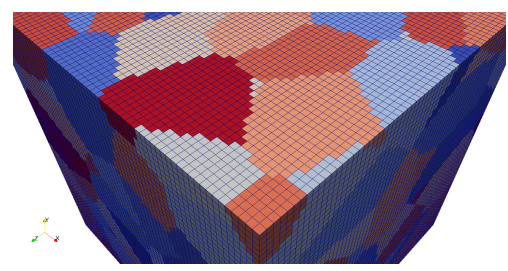

(d) Zoomed: $64^{3}$ voxels.

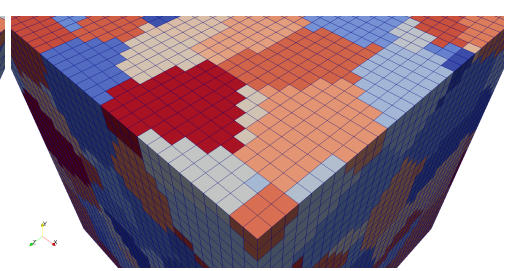

(c) Zoomed: $32^{3}$ voxels.

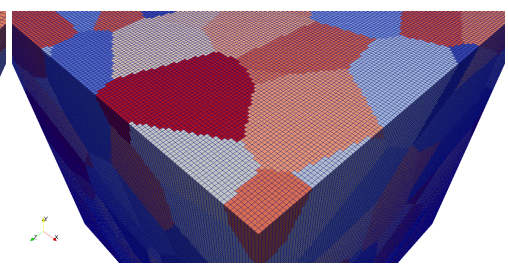

(e) Zoomed: $128^{3}$ voxels.

Figure 4: Periodic polycrystal aggregate models (100 grains) used in the CP-FFT simulations (discretization given in voxels). Note that same grain geometry was used as in Fig. 2.

$$
\operatorname{err}_{2}(k)=\frac{\left\|\left\langle\boldsymbol{\sigma}^{k}\right\rangle-\bar{k} \boldsymbol{\Sigma}^{o}\right\|}{\left\|\bar{k} \boldsymbol{\Sigma}^{o}\right\|}
$$

where $\left\langle\boldsymbol{\sigma}^{(k)}\right\rangle$ denotes the volume average of the stress at iterate $k$ and $\|\cdot\|$ denotes the Frobenius norms of a vector $\boldsymbol{v}$ or a second-order tensor $\boldsymbol{\tau}[28]$ :

$$
\|\boldsymbol{v}\|^{2}=\sum_{i=1,2,3} v_{i}^{2}, \quad\|\boldsymbol{\tau}\|^{2}=\tau_{i j} \tau_{i j}=\sum_{i=1,2,3} \sum_{j=1,2,3} \tau_{i j}^{2},
$$

and $\bar{k}$ is the unknown level of overall stress. The iterative procedure is stopped when the errors $\operatorname{err}_{1}$ and $\mathrm{err}_{2}$ are smaller than a prescribed value denoted by "TOL" in the following.

In all the simulations performed by the CP-FEM and CP-FFT (unless otherwise stated), the applied nominal strain rate $\dot{E}_{33}$ was set to $\dot{E}_{33}=310^{-4} \mathrm{~s}^{-1}$ and the simulations were terminated at a maximum nominal strain of $E_{33}=0.03$. Tables B.3 and B.4 in Appendix B list all the simulation cases studied with CP-FEM and CP-FFT, respectively.

To validate the implementations and assess the accuracy of the integration schemes in Abaqus and CraFT, a series of simple tensile tests was performed on single crystal aggregate models with various crystallographic orientations. An excellent agreement between the CP-FEM and CP-FFT results was found for all considered cases (not shown). The results were also checked to be independent of mesh density. It was therefore concluded that both methods as well as their implementations in Abaqus and CraFT are consistent with each other. 


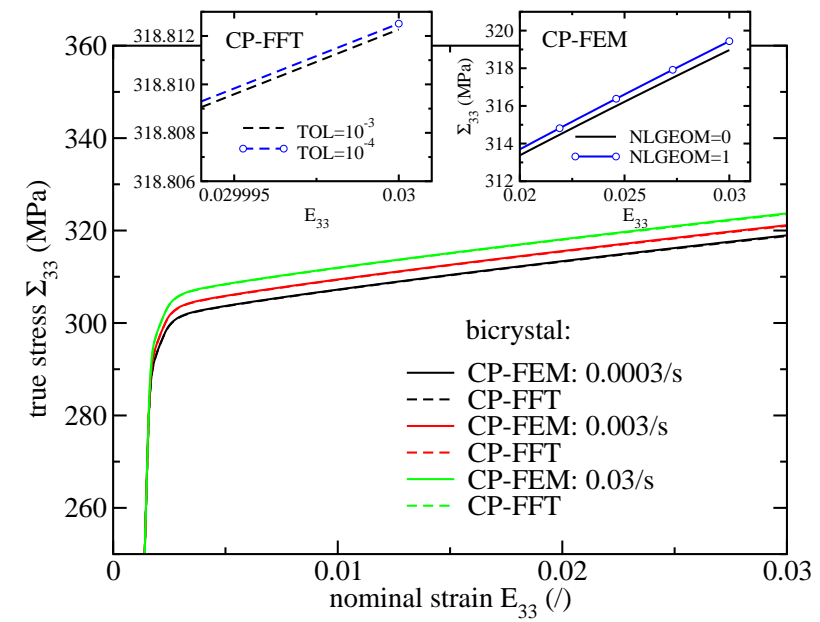

Figure 5: Bicrystal tensile curves calculated for three different strain rates (cases 9, 14, 15 in Tab. B.3 and cases 10, 12, 13 in Tab. B.4). A comparison is shown between the CP-FEM (full lines) and CP-FFT (dashed lines) simulations. Left inset shows the influence of the CP-FFT tolerance factor (cases 10 and 11 in Tab. B.4). Right inset shows the influence of geometric non-linearities on the CP-FEM results (cases 9 and 10 in Tab. B.3). Linear tetrahedral mesh was used for all CP-FEM results.

\section{Results and discussion}

\subsection{Bicrystal tensile response}

A bicrystal model (Fig. 1) was included in the study as the simplest topological realization that provides non-homogeneous stress/strain fields for the assumed uniaxial tension, thus providing a numerical playground for studying mesh convergence in both approaches. As it is shown later, our computational resources in fact allowed us to reach the converged limit of the model in terms of mesh density (defined as the number of elements/voxels per grain).

Figure 5 shows the calculated tensile responses of the bicrystal model, defined in Fig. 1, using three different strain rates. As expected, a stiffer response is observed for faster deformations. For each strain rate, the CP-FEM and CP-FFT curves overlap on the whole strain domain, again implying the equivalence of the two approaches. The insets of Fig. 5 moreover demonstrate the already established convergence of the CP-FFT results with respect to the tolerance factor and a small influence of the geometric non-linearities on the CP-FEM stresses at 0.03 nominal strain. This last observation also justifies the use of small strain approximation in this study.

Figure 6 presents mesh convergence analysis of the bicrystal model for various macroscopic quantities related to stress: the average Young modulus defined at 0.001 strain as $\mathrm{E}=\Sigma_{33}(0.001) / 0.001$, the yield stress calculated from the relation $\Sigma_{y}=\Sigma_{33}\left(E_{33, y}\right)=\mathrm{E}\left(E_{33, y}-0.002\right)$, and the final stress, $\Sigma_{0.03}=\Sigma_{33}(0.03)$. The results of Fig. 6 show a common trend: with increasing mesh density, all the considered quantities converge to common limiting values (note that smaller maximum densities could be reached with quadratic 


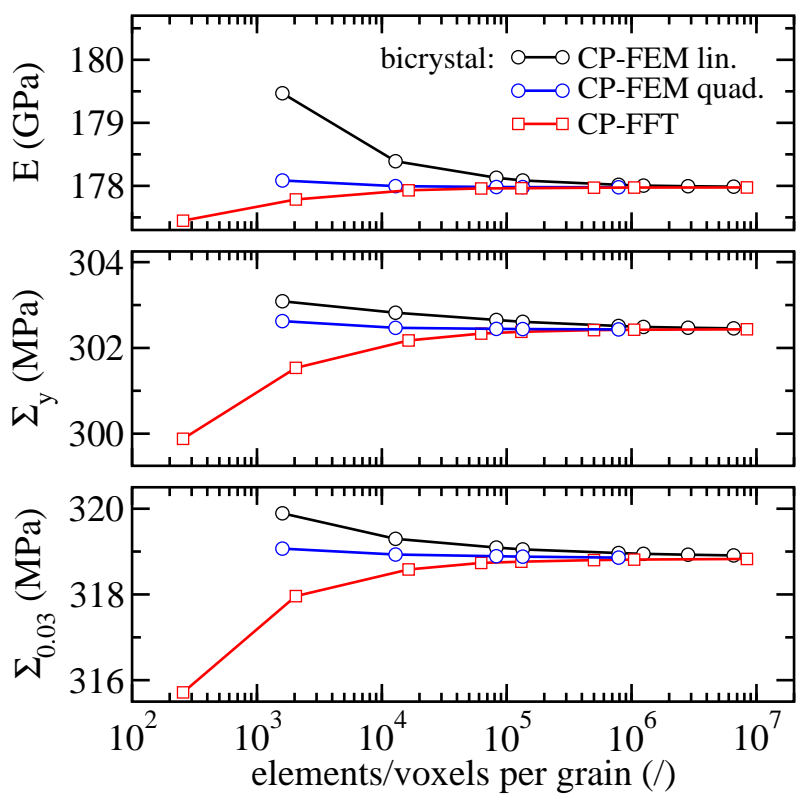

Figure 6: Mesh convergence analysis of various macroscopic quantities calculated in the bicrystal model. A comparison is shown between CP-FFT (squares) and CP-FEM (circles) simulations, using either linear (in black) or quadratic (in blue) tetrahedral mesh. See text for the definition of shown quantities. Strain rate: $310^{-4} / \mathrm{s}$.

CP-FEM meshes). Interestingly, as a general observation from Fig. 6, the average CP-FEM and CP-FFT results approach the limiting values from different directions: the CP-FEM from higher stress values, while the CP-FFT from lower stress values. A softer response of the CP-FEM model with more degrees of freedom per grain (higher mesh density) is well understood, however, the opposite behavior of the CP-FFT model is observed in the present context. Although not studied in detail, the observed behavior could be explained as an effect of the changing volume fraction of the grains in the CP-FFT model. Unlike the CP-FEM case where grain volumes are independent of mesh density, grain volumes in the CP-FFT model do change with varying spatial resolution. Changing volume fraction of the grains can affect the overall stiffness of the model resulting in different average stress values.

Results of Fig. 6 also indicate that CP-FFT and CP-FEM with linear tetrahedral elements demonstrate similar convergence rates with increasing mesh density. The fastest convergence in this respect is observed for CP-FEM quadratic mesh (see also Sec. 3.2 where the efficiency comparison of the three approaches is discussed in terms of CPU time).

In a similar fashion, Fig. 7 shows mesh convergence analysis of grain-averaged stresses calculated using element/voxel volume weighting, $\left\langle\sigma_{j j}\right\rangle_{k}=\sum_{i} V_{i} \sigma_{i, j j} / \sum_{i} V_{i}$, over all elements/voxels of grain $k$. A very good agreement is observed between CP-FEM and CP-FFT approaches, all reaching common limiting values at largest mesh densities (note that due to uniaxial loading, both $\left\langle\sigma_{11}\right\rangle_{1}=-\left\langle\sigma_{11}\right\rangle_{2}$ and $\left\langle\sigma_{22}\right\rangle_{1}=-\left\langle\sigma_{22}\right\rangle_{2}$ are exactly evaluated for all discretizations). A slightly slower mesh convergence is observed for CP-FEM results 

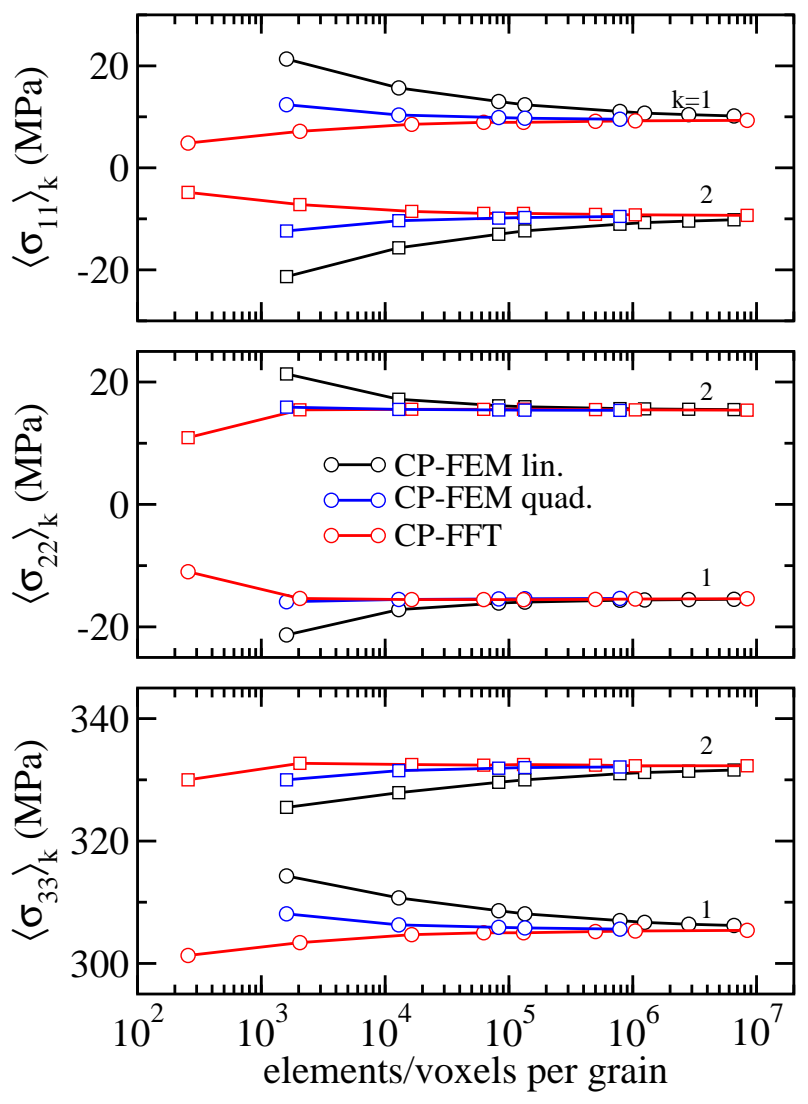

Figure 7: Mesh convergence analysis of grain-averaged stresses $\left\langle\sigma_{j j}\right\rangle_{k}$ calculated at 0.03 nominal strain in grain $k$ of the bicrystal model. A comparison is shown between the CP-FFT (in red) and CP-FEM simulations, using either linear (in black) or quadratic (in blue) tetrahedral mesh. See text for the definition of average stresses. Strain rate: $310^{-4} / \mathrm{s}$.

obtained on linear meshes, while CP-FFT results and CP-FEM results obtained on quadratic meshes seem to provide a similar, faster convergence rate, especially at lower spatial resolutions.

As a main result of this section, Fig. 8 presents mesh convergence analysis of average grain boundary normal stresses, $\left\langle\sigma_{n n}\right\rangle_{12}$, calculated on five different interfaces (grain boundaries) of the periodic bicrystal model. The periodicity of the bicrystal model allows one to study several (in this case up to eight) interfaces between the two grains (labeled 1 and 2) of the bicrystal model of Fig. 1(a). Each interface is further characterized by inclination angle $\theta$ between the loading tensile direction ( $z$ axis) and grain boundary normal $n$

While the introduced quantities, $\mathrm{E}, \Sigma_{y}$ and $\Sigma_{0.03}$ of Fig. 6 were calculated unambiguously from the simulated tensile curves, the calculations of $\left\langle\sigma_{n n}\right\rangle_{12}$ followed two slightly different approaches as described below.

In the CP-FEM approach, the grain boundary geometry was exactly resolved by tetrahedral elements. 

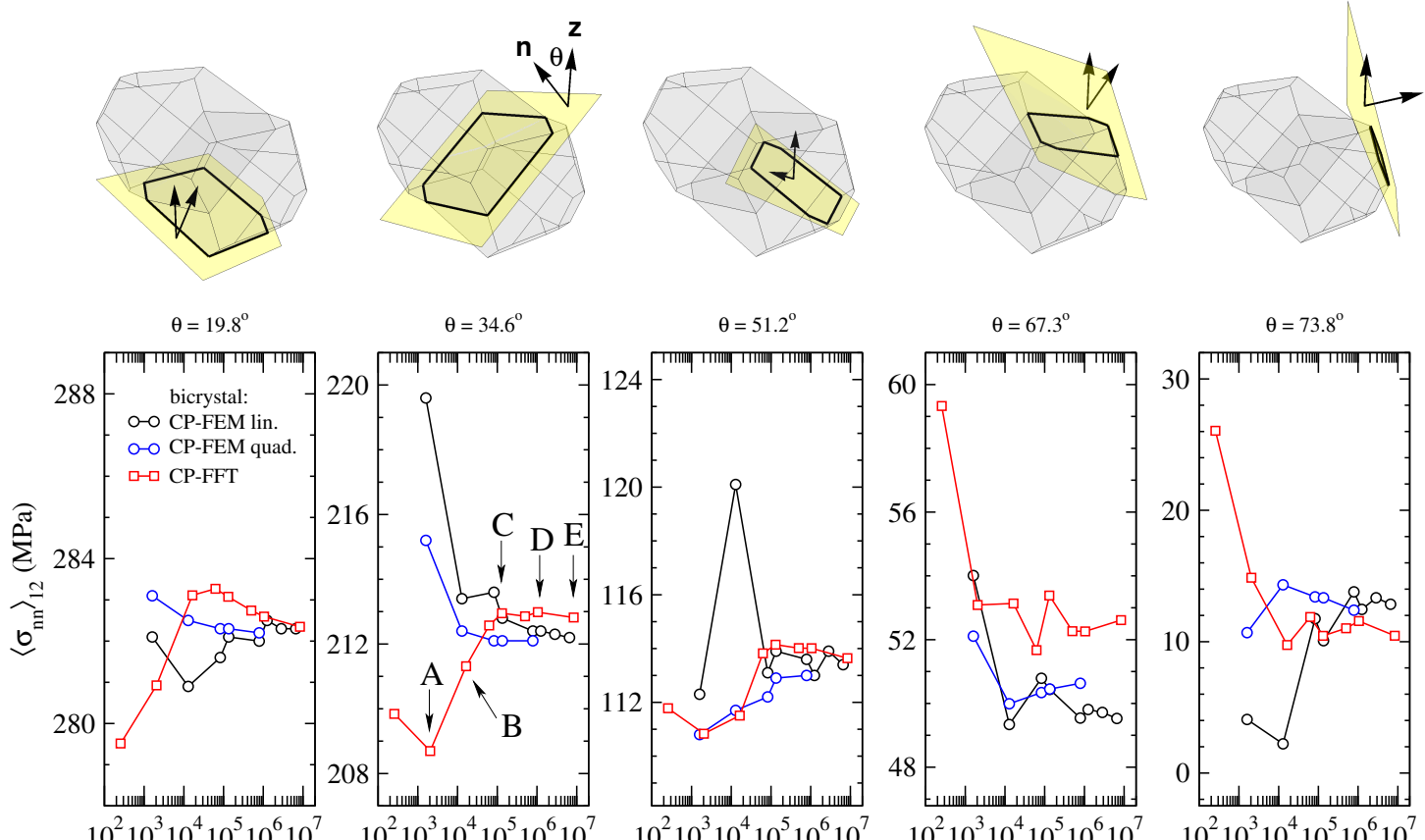

elements/voxels per grain (/)

Figure 8: Mesh convergence analysis of average grain boundary normal stresses calculated on five different interfaces of the bicrystal model denoted by angle $\theta$ between the loading direction ( $z$ axis) and grain boundary normal $\boldsymbol{n}$ (see sketches above). A comparison is shown between CP-FFT (squares) and CP-FEM (circles) simulations, using either linear (in black) or quadratic (in blue) tetrahedral mesh. Letters A, B, C, D, E denote mesh discretizations for which $\sigma_{n n}$ field maps are compared in Fig. 9. All $\left\langle\sigma_{n n}\right\rangle_{12}$ were calculated at 0.03 nominal strain. Strain rate: $310^{-4} / \mathrm{s}$.

For each pair $i$ of tetrahedral elements touching the particular grain boundary ${ }^{3}$, two Cauchy stresses, $\boldsymbol{\sigma}_{i, 1}$ and $\boldsymbol{\sigma}_{i, 2}$, in the case of C3D4 elements and respectively six Cauchy stresses, $\boldsymbol{\sigma}_{i, 1} \ldots \boldsymbol{\sigma}_{i, 6}$, in the case of quadratic C3D10 elements were obtained at the corresponding Gauss points located closest to the interface. These were then used to calculate one single value for the normal stress per element pair, $\sigma_{i, n n}=1 / 2 \sum_{j=1}^{2} \boldsymbol{n}_{i} . \boldsymbol{\sigma}_{i, j} . \boldsymbol{n}_{i}$ in the case of linear elements and $\sigma_{i, n n}=1 / 6 \sum_{j=1}^{6} \boldsymbol{n}_{i} . \boldsymbol{\sigma}_{i, j} . \boldsymbol{n}_{i}$ in the case of quadratic elements, respectively, knowing the normal $\boldsymbol{n}_{i}$ of the grain boundary facet $i$ (in fact, stress projections were performed in the undeformed configuration, thus $\boldsymbol{n}_{i}=\boldsymbol{n}$ ). As elements of different size were used in the finite element mesh, the occurrence of $\sigma_{i, n n}$ in the computation of the average grain boundary normal stress was weighted by the surface $A_{i}$ of the grain boundary facet on which it was obtained, $\left\langle\sigma_{n n}\right\rangle=\sum_{i} A_{i} \sigma_{i, n n} / \sum_{i} A_{i}$. Note that same approach was used in $[9,10]$.

In the CP-FFT approach, a discretization of the modeling space into voxels led to approximate step-

\footnotetext{
${ }^{3}$ Tetrahedral element can touch grain boundary either with its facet, edge or node. In this study, only pairs $i$ of tetrahedra are considered which share common grain boundary facet. In this case the associated facet area $A_{i}$ and normal $\boldsymbol{n}_{i}$ are well defined.
} 
like grain boundaries. To calculate $\left\langle\sigma_{n n}\right\rangle$ for one particular grain boundary, first all voxels $i$ containing the analytical grain boundary plane (with corresponding normal $\boldsymbol{n}$ ) were identified along with the corresponding stresses $\boldsymbol{\sigma}_{i}$ and areas $A_{i}$ of the cross-sections between the voxel $i$ and analytical grain boundary plane. For each such voxel, a grain boundary normal stress was then calculated as $\sigma_{i, n n}=\boldsymbol{n} . \boldsymbol{\sigma}_{i} . \boldsymbol{n}$. Similarly to the CP-FEM procedure, the computation of the average grain boundary normal stress was finally obtained by surface weighting, $\left\langle\sigma_{n n}\right\rangle=\sum_{i} A_{i} \sigma_{i, n n} / \sum_{i} A_{i}$.

Results of Fig. 8 show convergence behavior of $\left\langle\sigma_{n n}\right\rangle_{12}$ with increasing spatial resolution for all five considered grain boundary interfaces of the bicrystal model and for all three applied approaches. However, in practically all considered cases the observed evolutions of $\left\langle\sigma_{n n}\right\rangle_{12}$ are not so smooth as in Figs. 6 and 7 : significant oscillations (fluctuations) of $\left\langle\sigma_{n n}\right\rangle_{12}$ may be attributed to a generally smaller averaging sample size (surface averaging instead of volume averaging). Convergence rates of CP-FEM with quadratic elements (and to some extend also linear elements) and CP-FFT seem to be comparable at a given inclination $\theta$. However, the agreement between the CP-FEM and CP-FFT limiting values seems to be better at smaller (and intermediate) inclinations $\theta$, with discrepancies smaller than $\sim 1 \mathrm{MPa}$. At higher angles $\theta$ the differences increase but seemingly do not exceed $\sim 4 \mathrm{MPa}$. The observed (small) disagreement between CP-FEM and CP-FFT limiting values for $\left\langle\sigma_{n n}\right\rangle_{12}$ may be attributed to slightly different approaches used in the calculation of $\left\langle\sigma_{n n}\right\rangle_{12}{ }^{4}$.

Finally, a comparison of local grain boundary normal stresses, calculated on the largest grain boundary interface of the bicrystal model, is presented in Fig. 9 for all three applied methods and various spatial discretizations. In the limit of finest mesh discretizations all three methods provide very similar $\sigma_{n n}$ maps. Nevertheless, the best performance in terms of locally converged $\sigma_{n n}$ may be assigned to quadratic CP-FEM mesh which shows stable and smooth evolution of local stresses without any spurious stress fluctuations. These are, however, visible in both CP-FFT and linear mesh CP-FEM. While checkerboard-like fluctuations of stresses in the later case can be attributed partly to volumetric locking of overly-stiff C3D4 elements, the spurious oscillations observed in CP-FFT maps are a well-known drawback of the spectral methods [29]; however, these oscillations are small and can be further reduced using different filtering techniques [29]. Although $\sigma_{n n}$ values were calculated differently for CP-FEM and CP-FFT models (see discussion on $\left\langle\sigma_{n n}\right\rangle$ ), both procedures seem to be robust enough to provide local stress maps consistent with each other. The comparison in Fig. 9 therefore confirms that CP-FFT model discretization into regular voxel grid is suitable to provide accurate local stresses on otherwise approximately resolved grain boundaries.

Finally, the influence of simulation time step and time integration scheme of the material model is analyzed in more detail in Appendix C.

\footnotetext{
${ }^{4} \mathrm{~A}$ possible source of the observed disagreement in the calculated $\left\langle\sigma_{n n}\right\rangle_{12}$ may also be due to the omission of tetrahedral elements in the CP-FEM approach that touch the grain boundary with a single node or single edge.
} 


\subsection{Polycrystal tensile response}

In this section, the equivalence of the three approaches demonstrated in the bicrystal case is challenged in the more realistic 100-grain polycrystalline model of stainless steel irradiated to 0.8 dpa, see Fig. 2 . Similarly to the bicrystal case, periodic grain geometry, periodic boundary conditions and uniaxial tensile loading were applied to the model, however, random grain orientations were assumed to provide zero overall crystallographic texture. In the following, similar strategy as in bicrystal case is employed to compare the CP-FEM (using linear and quadratic meshes) and CP-FFT approaches.

Figure 10 presents the calculated tensile responses of the polycrystal model for three different strain rates and for 13777 and 20972 elements/voxels per grain (for CP-FEM and CP-FFT, respectively). Although the effect of strain rate is visible, being of the same order as in the bicrystal case, a much stronger stress gap is observed between the CP-FEM (using linear tetrahedral mesh) and CP-FFT responses in the whole plastic regime. The gap opens at yield strain and grows with the increasing strain. The insets of Fig. 10 clearly demonstrate that discrepancies between the linear mesh CP-FEM and CP-FFT results are not due to insufficient tolerance factor, simulation time step nor geometric non-linearities. In fact, the observed differences are attributed to reduced performance of linear tetrahedral elements C3D4, showing overly stiff response. For example, a substantially softer response is observed for quadratic tetrahedral mesh shown in the right inset of Fig. 10.

The properties of the gap are discussed further in Fig. 11 through mesh convergence analysis of E, $\Sigma_{y}$ and $\Sigma_{0.03}$ which were extracted directly from the simulated tensile curves using the same definitions as before. In general, the same trend is observed as in the bicrystal case (Fig. 6), although not so dense meshes could be reached with the same computational resources (note that C3D4 and C3D10 meshes are topologically equivalent for a given number of elements, however smaller maximum mesh densities could be reached with C3D10 tetrahedra). While the values for E do converge to the same limit for both CP-FEM mesh types and CP-FFT, the involvement of plasticity (lower two panels in Fig. 11) makes the convergence of the linear mesh CP-FEM much slower. In fact, it seems that convergence rate of linear mesh CP-FEM results decreases with increasing (plastic) strain, while the convergence rates of quadratic mesh CP-FEM and CP-FFT results are approximately strain independent (although a much smaller decrease of convergence rate can be also seen for quadratic mesh CP-FEM results at 0.03 nominal strain).

This is indeed confirmed in Fig. 12 where relative quantities are compared for increasing mesh density to better facilitate the convergence rate as a function of applied strain (elastic strain - top panel, yield strain - middle panel, maximum strain - bottom panel). For comparison, the results of the bicrystal case are also included, showing practically no influence of strain on the convergence rate for both CP-FEM (regardless of mesh type) and CP-FFT approaches. It is positively surprising, though, that the CP-FFT convergence rates for $\mathrm{E}, \Sigma_{y}$ and $\Sigma_{0.03}$ are not only independent of strain but also of the number of grains employed in the model. This aggregate-size invariance makes the CP-FFT approach clearly superior to both CP-FEM 
approaches. Namely, at 0.03 nominal strain (bottom panel), the convergence rate of the quadratic mesh CP-FEM polycrystal is clearly smaller than that of the bicrystal model.

The comparison between the three approaches is continued in Fig. 13 by analyzing mesh convergence of grain-averaged axial stresses $\left\langle\sigma_{33}\right\rangle_{k}$ and grain-boundary-averaged normal stresses $\left\langle\sigma_{n n}\right\rangle_{i j}$ at 0.03 nominal strain. Similar relative behavior is observed for $\left\langle\sigma_{33}\right\rangle_{k}$ as in Fig. 11: linear mesh CP-FEM results seem to be over-predicted, demonstrating much slower mesh convergence than quadratic mesh CP-FEM and CP-FFT results. As there are many grain boundaries available in a 100-grain polycrystal, only three of them were selected for the mesh convergence analysis that provided highest $\left\langle\sigma_{n n}\right\rangle_{i j}$ values at the maximum applied strain 0.03. It is believed $[9,10]$ that such grain boundaries are potentially most dangerous spots inside a stainless steel material for developing intergranular cracks in the presence of corrosive environment (IASCC). The results presented in the bottom panel of Fig. 13 show that largest values of $\left\langle\sigma_{n n}\right\rangle_{i j}$ exceed substantially (up to $\sim 40 \%$ ) the macroscopic tensile stress ( $\sim 400 \mathrm{MPa}$, see $\Sigma_{0.03}$ in Fig. 11). Similar to $\Sigma_{0.03},\left\langle\sigma_{n n}\right\rangle_{i j}$ also seem to be overestimated in the linear mesh CP-FEM which is furthermore supported by the fact that the CP-FFT results converge faster and more smoothly with the increasing mesh density. The convergence of the quadratic mesh CP-FEM results seems to be comparable to that of the CP-FFT.

In Fig. 14 a more detailed analysis of grain boundary normal stresses $\sigma_{n n}$ is finally shown for one particular grain boundary located between grains 9 and 70. This grain boundary provides the largest (tensile) average normal stress $\left\langle\sigma_{n n}\right\rangle_{9-70}$. Note that the normal of this grain boundary is practically aligned with the tensile direction ( $z$ axis). Although the grain boundary geometry is approximately resolved within the CP-FFT approach, the consistency of the calculated $\sigma_{n n}$ field is obvious: the amplitudes and locations of the maximum/minimum $\sigma_{n n}$ stresses are more or less independent of mesh density (even on the coarsest mesh), thus implying a very good convergence rate not only of the average stress $\left\langle\sigma_{n n}\right\rangle_{i j}$ but also of local $\sigma_{n n}$ fields. On the contrary, the linear mesh CP-FEM results show considerably higher fluctuations of $\sigma_{n n}$ with increasing mesh density. The maximum/minimum $\sigma_{n n}$ values not only change in amplitude but also appear at different locations of the grain boundary. Similarly, stress fluctuations observed on quadratic mesh CP-FEM still persist but with smaller extent.

Note, however, that practically same $\sigma_{n n}$ fields were obtained in the bicrystal case in the limit of highest mesh densities (see Fig. 9). It is therefore reasonable to conclude that CP-FFT approach performs better than linear/quadratic mesh CP-FEM also when considering local grain boundary stresses in larger aggregates.

The results so far have shown a superior behavior of the CP-FFT approach in terms of mesh convergence. One of major reasons to use CP-FFT method is also its superior computational performance. In this respect, the efficiency comparison of the three approaches was performed in terms of CPU time by running both codes (CraFT and Abaqus) on the same computer cluster. A comparison was performed on a 100-grain case with 125k voxels and 121k elements using both C3D4 and C3D10 meshes. These models were selected 


\begin{tabular}{lc}
\hline \hline Model & total CPU time $(\mathrm{h})$ \\
\hline CP-FFT & 3.4 \\
CP-FEM lin. & 30 \\
CP-FEM quad. & 210 \\
\hline \hline
\end{tabular}

Table 2: A comparison of simulation CPU times for three different models: a 100-grain case with 125k voxels (CP-FFT) and $121 \mathrm{k}$ elements using either linear (CP-FEM lin.) or quadratic elements (CP-FEM quad.). All computations were performed on a single computational node using parallelization on 20 processors.

as they share a similar number of voxels/elements. The simulation CPU times are summarized in Tab. 2, showing a superior efficiency behavior of CP-FFT in comparison to CP-FEM.

\section{Conclusions}

Crystal plasticity constitutive laws for the irradiated austenitic stainless steel SA304L were implemented within the finite element (CP-FEM) and FFT-based spectral density (CP-FFT) frameworks using Abaqus and CraFT codes, respectively. The performance of the two approaches was analyzed in terms of convergence rates of various macroscopic stress quantities calculated in non-trivial periodic Voronoi aggregates with different spatial discretizations. In CP-FEM analyses linear and quadratic tetrahedral elements were used to mesh complex grain geometries. The following conclusions were identified:

- The evolution of the macroscopic stress quantities with increasing mesh density showed two opposite trends: the CP-FEM results on linear and quadratic meshes got softer while the CP-FFT results got stiffer with finer mesh discretization.

- Within the studied bicrystal model, the CP-FEM and CP-FFT results were shown to converge to same limiting values with similar convergence rates, thus further demonstrating the equivalence of the two approaches in aggregates with relatively small number of grains.

- The performance of the two approaches diverged in the 100-grain polycrystalline model: while the convergence rate of the CP-FFT results was practically independent of the applied strain as well as the number of grains employed in the model, much slower convergence of the linear mesh CP-FEM results with increasing mesh density was observed at higher plastic strains and for larger aggregates. The same trend but with reduced extent was observed also in quadratic mesh CP-FEM results.

- Similar conclusion was shown to hold for local and average grain boundary stresses: the linear mesh CP-FEM approach overestimated the highest intergranular normal stresses, thus raising a concern of possible over-conservatism when predicting stress-corrosion-cracking (IASCC) initiation with the CP-FEM in irradiated austenitic stainless steels. 
- The observed aggregate-size invariance of the mesh convergence rate makes the CP-FFT approach superior to the CP-FEM, especially in larger aggregates when deformed to higher (plastic) strains.

- CP-FFT approach demonstrates also superior computational efficiency in terms of simulation CPU time: CP-FFT simulations are approximately ten (sixty) times faster than equivalent simulations performed on linear (quadratic) CP-FEM meshes.

\section{Acknowledgments}

The authors acknowledge the financial support from European Committee through the SOTERIA H2020 research project funded under the Euratom research and training programme 2014-2018 under grant agreement $\mathrm{N}^{\circ} 661913$.

\section{Data availability}

The raw and processed data required to reproduce these findings are available from the corresponding author upon reasonable request.

\section{Appendix A. Small strain formulation of crystal plasticity model}

The main equations of the small strain formulation of the crystal plasticity model are written below,

$$
\dot{\sigma}=\mathbb{C}:\left(\dot{\boldsymbol{\epsilon}}-\dot{\boldsymbol{\epsilon}}^{v p}\right)
$$

where the second-order tensors $\boldsymbol{\sigma}, \boldsymbol{\epsilon}, \boldsymbol{\epsilon}^{v p}$ denote the stress, total strain, and viscoplastic strain (a dot over a variable denotes its time derivative). The fourth-order elastic stiffness tensor is denoted by $\mathbb{C}$. The viscoplastic strain rate writes

$$
\dot{\boldsymbol{\epsilon}}^{v p}=\sum_{\alpha=1}^{12} \dot{\gamma}^{\alpha} \boldsymbol{\mu}^{\alpha},
$$

where $\gamma^{\alpha}$ is the shear strain in slip system $\alpha\left(\alpha=1 \ldots 12\right.$ for face-centered-cubic lattice) and $\boldsymbol{\mu}^{\alpha}$ is the usual Schmid tensor in slip system $\alpha$. The resolved shear stress $\tau^{\alpha}$, entering the shear flow law of Eq. (1), is defined as

$$
\tau^{\alpha}=\boldsymbol{\sigma}: \boldsymbol{\mu}^{\alpha} .
$$

The parameters entering this crystal plasticity law are described in Sec. 2 together with the critical resolved shear stress and the evolution laws for the normalized dislocation and Frank loop densities, Eqs. (2), (3) and (4), respectively. 


\section{Appendix B. Simulation cases}

Tables B.3 and B.4 list all the simulation cases studied in this work.

\section{Appendix C. Influence of simulation time step and time integration scheme}

In this section, the influence of simulation time step on various macroscopic properties is analyzed and compared between CP-FEM and CP-FFT approaches. In addition, the effect of using a fully-implicit time integration scheme for the material CP-FEM model is briefly studied in the context of the results of the semi-implicit scheme.

Figure C.15 shows the influence of simulation time step $\Delta t$ of the time integration scheme on the various quantities introduced in Fig. 6. As expected, the results tend to converge with decreasing $\Delta t$, reaching reasonably well converged results for $\Delta t=0.1 \mathrm{~s}$ for both CP-FEM and CP-FFT simulations. Note that $\Delta t=0.1 \mathrm{~s}$ corresponds to 1000 increments within a total time window of $100 \mathrm{~s}$ needed to reach nominal strain of 0.03 with a given strain rate $310^{-4} / \mathrm{s}$.

As indicated before in Sec. 2.1, a semi-implicit time integration scheme was used in all CP-FEM simulations to facilitate faster computations. In order to provide a fair comparison with the CP-FFT approach, a fully-implicit time integration scheme was adopted for one particular CP-FEM case as illustrated in Fig. C.15 (star symbols). The differences arising due to using different integration schemes increase with the applied strain (top two panels for elastic strain, bottom two panels for 0.03 strain) but nevertheless remain negligibly small, with maximum relative error smaller than $5 \times 10^{-5}$. Such small differences seem to justify the use of a quicker semi-implicit time integration scheme.

\section{References}

[1] INTERnAtionAl ATOMIC ENERGY AGENCY, Assessment and Management of Ageing of Major Nuclear Power Plant Components Important to Safety: PWR Vessel Internals, INTERNATIONAL ATOMIC ENERGY AGENCY, Vienna, 1999, IAEA-TECDOC-1119.

[2] O. K. Chopra, A. S. Rao, A review of irradiation effects on LWR core internal materials neutron embrittlement, Journal of Nuclear Materials 412 (1) (2011) 195 - 208. doi:https://doi.org/10.1016/j.jnucmat.2011.02.059.

[3] O. K. Chopra, A. S. Rao, A review of irradiation effects on LWR core internal materials IASCC susceptibility and crack growth rates of austenitic stainless steels, Journal of Nuclear Materials 409 (3) (2011) 235 - 256. doi:https://doi.org/10.1016/j.jnucmat.2010.12.001.

[4] Y. Chen, A. Rao, B. Alexandreanu, K. Natesan, Slow strain rate tensile tests on irradiated austenitic stainless steels in simulated light water reactor environments, Nuclear Engineering and Design 269 (2014) 38 - 44, special Issue - The International Conference on Structural Mechanics in Reactor Technology (SMiRT21), New Delhi India, Nov 06-11, 2011. doi:https://doi.org/10.1016/j.nucengdes.2013.08.003.

[5] N. Barton, J. Arsenlis, A. ans Marian, A polycrystal plasticity model of strain localization in irradiation iron, J. Mech. Phys. Solids 61 (2013) 341-351. 
[6] X. Han, Modélisation de la fragilisation due au gonflement dans les aciers inoxydables austénitiques irradiés, Ph.D. thesis, Ecole Nationale Supérieure des Mines de Paris (2012).

[7] B. Tanguy, X. Han, J. Besson, S. Forest, C. Robertson, N. Rupin, Dislocations and irradiation defects-based micromechanical modelling for neutron irradiated austenitic stainless steels, in: International Symposium on Plasticity 2013 and Its Current Applications, January 3-8, 2013, Nassau, Bahamas, 2013.

[8] G. Monnet, C. Mai, Prediction of irradiation hardening in austenitic stainless steels: Analytical and crystal plasticity studies, J. Nuc. Mat. 518 (2019) 316-325.

[9] J. Hure, S. El Shawish, L. Cizelj, B. Tanguy, Intergranular stress distributions in polycrystalline aggregates of irradiated stainless steel, J. Nuc. Mat. 476 (2016) 231-242.

[10] S. El Shawish, J. Hure, Intergranular normal stress distributions in untextured polycrystalline aggregates, Eur. J. Mech. / A Solid 72 (2018) 354-373.

[11] Simulia, ABAQUS 6.14-2 (2016).

[12] P. Suquet, H. Moulinec, O. Castelnau, M. Montagnat, N. Lahellec, F. Grennerat, P. Duval, R. Brenner, Multiscale modeling of the mechanical behavior of polycrystalline ice under transient creep, Procedia IUTAM 3 (2012) 76 - 90, IUTAM Symposium on Linking Scales in Computations: From Microstructure to Macro-scale Properties. doi:https://doi.org/10.1016/j.piutam.2012.03.006.

[13] B. Liu, D. Raabe, F. Roters, P. Eisenlohr, R. A. Lebensohn, Comparison of finite element and fast fourier transform crystal plasticity solvers for texture prediction, Modelling and Simulation in Materials Science and Engineering 18 (8).

[14] P. Eisenlohr, M. Diehl, R. Lebensohn, F. Roters, A spectral method solution to crystal elasto-viscoplasticity at finite strains, International Journal of Plasticity 46 (2013) 37 - 53, microstructure-based Models of Plastic Deformation. doi:https://doi.org/10.1016/j.ijplas.2012.09.012.

[15] C. Robert, C. Mareau, A comparison between different numerical methods for the modeling of polycrystalline materials with an elasticviscoplastic behavior, Computational Materials Science 103 (2015) 134 - 144. doi:https://doi.org/10.1016/j.commatsci.2015.03.028.

[16] S. Lucarini, J. Segurado, On the accuracy of spectral solvers for micromechanics based fatigue modeling, Computational Mechanics 63 (2) (2019) 365-382. doi:10.1007/s00466-018-1598-1.

[17] M. Victoria, N. Baluc, C. Bailat, Y. Dai, M. I. Luppo, R. Schublin, B. N. Singh, The microstructure and associated tensile properties of irradiated fcc and bcc metals, J. Nucl. Mat. 276 (2000) 114-122.

[18] S. Krishna, S. De, A temperature and rate-dependent micromechanical model of molybdenum under neutron irradiation, Phil. Mag. 90 (2010) 4013-4025.

[19] S. El Shawish, L. Cizelj, B. Tanguy, X. Han, J. Hure, Extended crystal plasticity finite element approach for neutron irradiated austenitic stainless steels, in: 23rd International Conference Nuclear Energy for New Europe, September 8-11, 2014, Portorož, Slovenia, 2014.

[20] L. Joëssel, Micromechanical modeling of the viscoplastic behavior of a porous polycrystal: application to an irradiated austenitic stainless steel, Theses, Université d'Aix-Marseille (2018).

[21] R. Quey, P. R. Dawson, F. Barbe, Large-scale 3d random polycrystals for the finite element method: Generation, meshing and remeshing, Comput. Methods Appl. Mech. Eng. 200 (2011) 1729-1745.

[22] G. Boittin, P.-G. Vincent, H. Moulinec, M. Gărăjeu, Numerical simulations and modeling of the effective plastic flow surface of a biporous material with pressurizedintergranular voids, Computer Methods in Applied Mechanics and Engineering 323 (2017) 174 - 201. doi:https://doi.org/10.1016/j.cma.2017.05.004.

[23] P.-G. Vincent, P. Suquet, Y. Monerie, H. Moulinec, Effective flow surface of porous materials with two populations of voids under internal pressure: II. full-field simulations, International Journal of Plasticity 56 (2014) 74 - 98. doi:https://doi.org/10.1016/j.ijplas.2013.11.012. 
[24] J. Michel, H. Moulinec, P. Suquet, Effective properties of composite materials with periodic microstructure: a computational approach, Computer Methods in Applied Mechanics and Engineering 172 (1) (1999) 109 - 143. doi:https://doi.org/10.1016/S0045-7825(98)00227-8.

[25] H. Moulinec, P. Suquet, A fast numerical method for computing the linear and nonlinear properties of composites, C. R. Acad. Sc. Paris II 318 (1994) 1417-1423.

[26] H. Moulinec, P. Suquet, A numerical method for computing the overall response of nonlinear composites with complex microstructure, Comp. Meth. Appl. Mech. Engng. 157 (1998) 69-94.

[27] L. Joëssel, P.-G. Vincent, M. Gărăjeu, M. I. Idiart, Viscoplasticity of voided cubic crystals under hydrostatic loading, International Journal of Solids and Structures 147 (2018) 156 - 165. doi:https://doi.org/10.1016/j.ijsolstr.2018.05.022.

[28] K. Wojtacki, P.-G. Vincent, P. Suquet, H. Moulinec, G. Boittin, A micromechanical model for the secondary creep of elasto-viscoplastic porous materials with two rate-sensitivity exponents: Application to a mixed oxide fuel, International Journal of Solids and Structuresdoi:https://doi.org/10.1016/j.ijsolstr.2018.12.026.

[29] L. Gélébart, F. Ouaki, Filtering material properties to improve fft-based methods for numerical homogenization, J. Comp. Phys. 294 (2015) 90-95. 


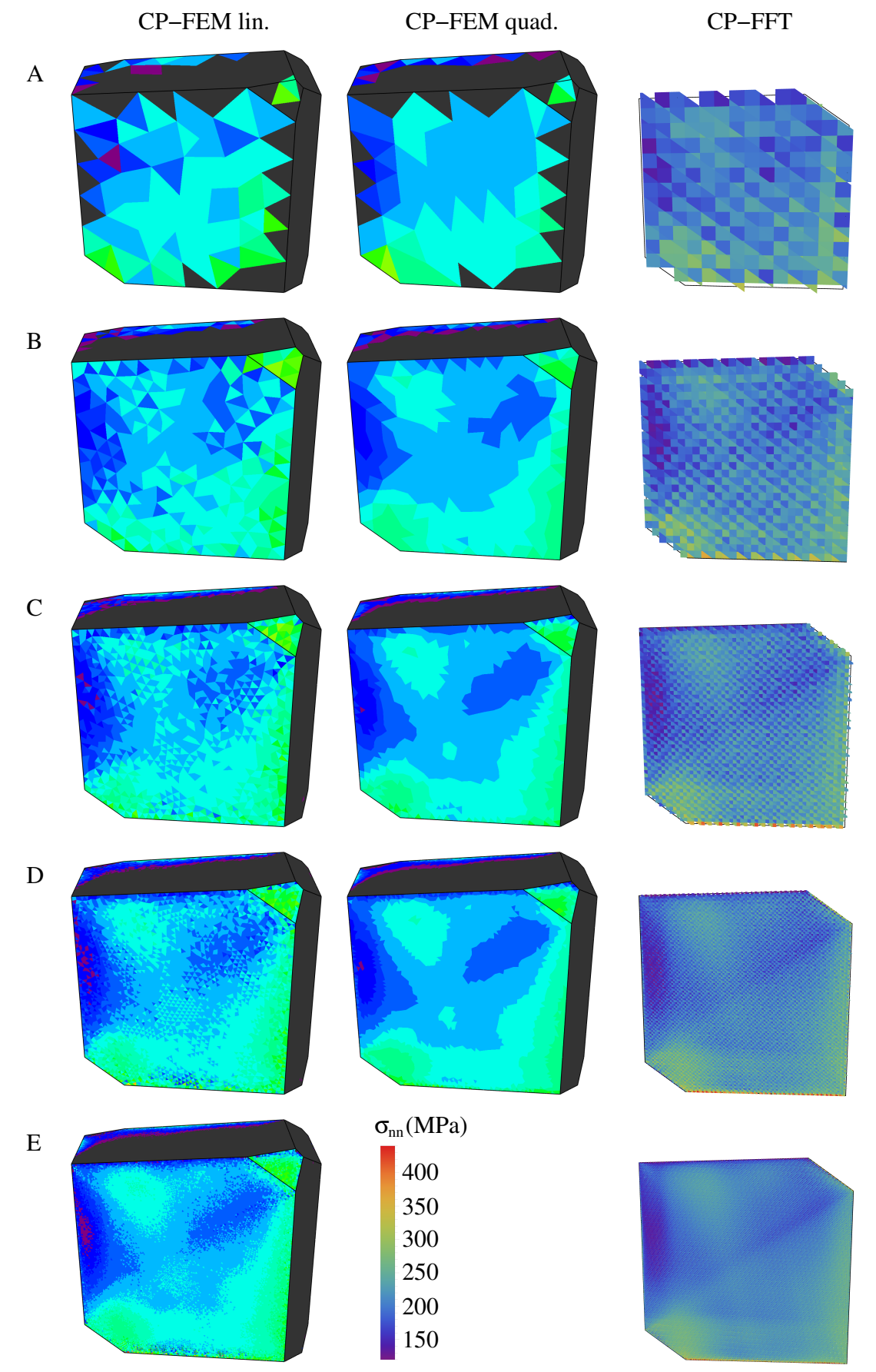

Figure 9: Maps of grain boundary normal stresses $\sigma_{n n}$ calculated on the largest grain boundary $\left(\theta=34.6^{\circ}\right)$ of the bicrystal model for different mesh discretizations denoted by letters A, B, C, D, E (see Fig. 8) at 0.03 nominal strain. No additional averaging was used in the presented maps (quilt-style contours). 


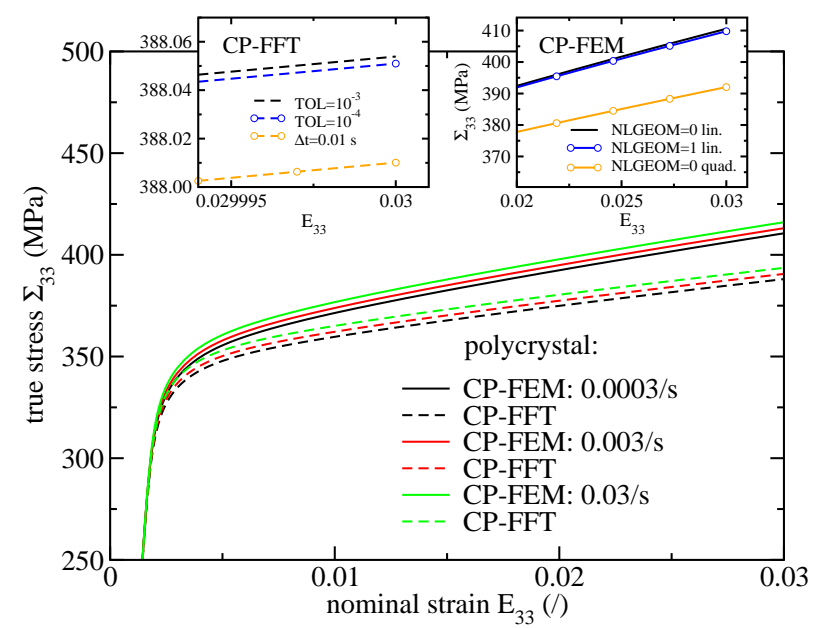

Figure 10: Polycrystal tensile curves calculated for 13777 elements per grain (CP-FEM) and 20972 voxels per grain (CP-FFT) and three different strain rates (cases 22, 24, 25 in Tab. B.3 and cases 21, 23, 24 in Tab. B.4). A comparison is shown between the CP-FEM (full lines) and CP-FFT (dashed lines) simulations. Linear tetrahedral mesh was used for CP-FEM results. Left inset shows the influence of the CP-FFT tolerance factor (cases 21 and 22 in Tab. B.4) and simulation time step (case 20 in Tab. B.4). Right inset shows the influence of geometric non-linearities and quadratic tetrahedral mesh on the CP-FEM results (cases 22 and 23 in Tab. B.3).

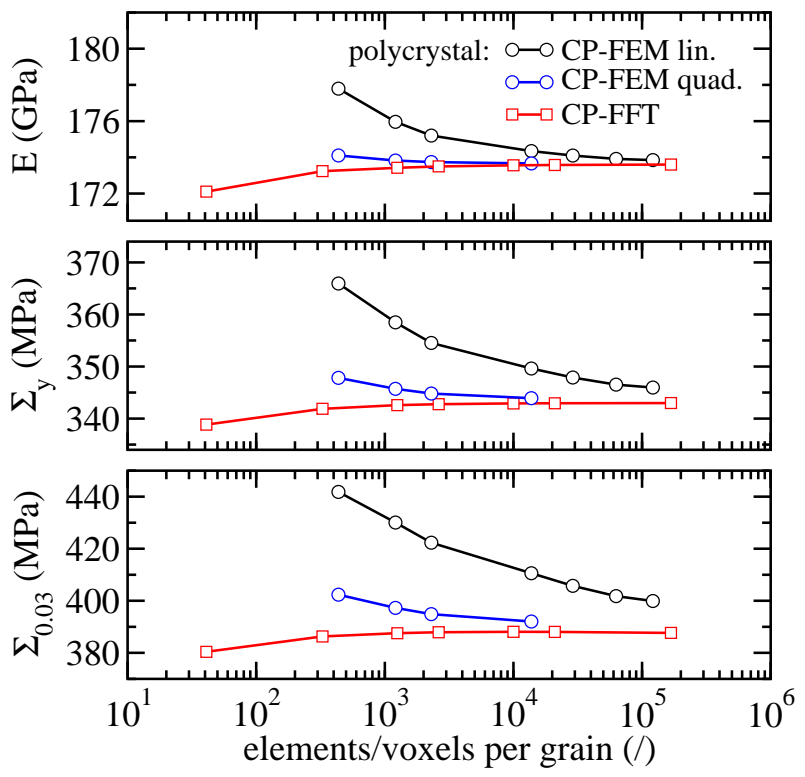

Figure 11: Mesh convergence analysis of various macroscopic quantities calculated in the polycrystal model. A comparison is shown between CP-FFT (squares) and CP-FEM (circles) simulations, using either linear (in black) or quadratic (in blue) tetrahedral mesh. Strain rate: $310^{-4} / \mathrm{s}$. 


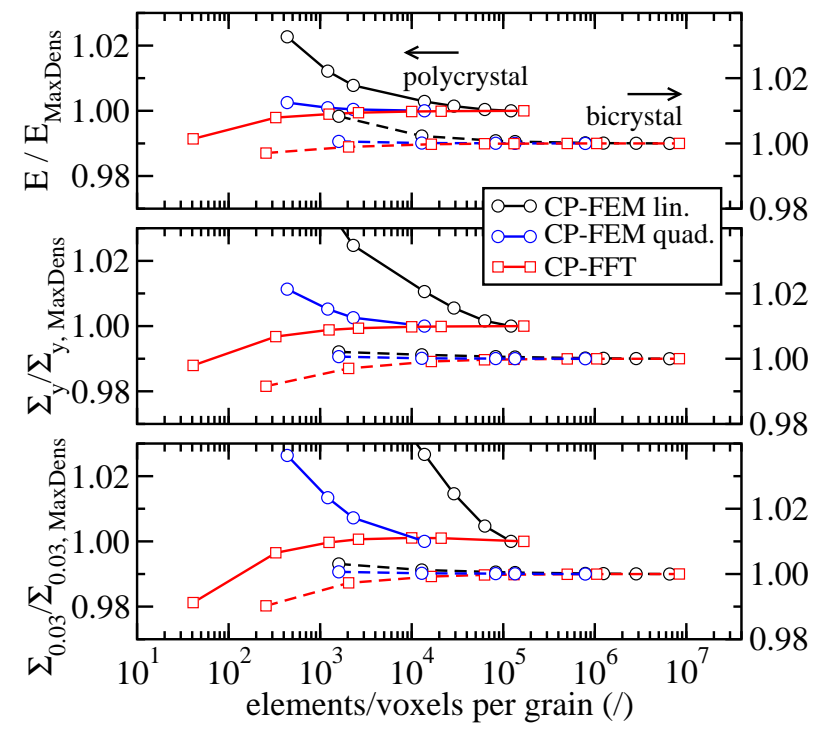

Figure 12: Mesh convergence rate analysis of various relative macroscopic quantities. Subscript MaxDens denotes the value obtained for the densest mesh. A comparison is shown between CP-FFT (squares) and CP-FEM (circles) simulations, using either linear (in black) or quadratic (in blue) tetrahedral mesh, performed in the bicrystal (dashed lines) and polycrystal (full lines) models. Note different vertical scales for bicrystal and polycrystal results. Strain rate: $310^{-4} / \mathrm{s}$. 

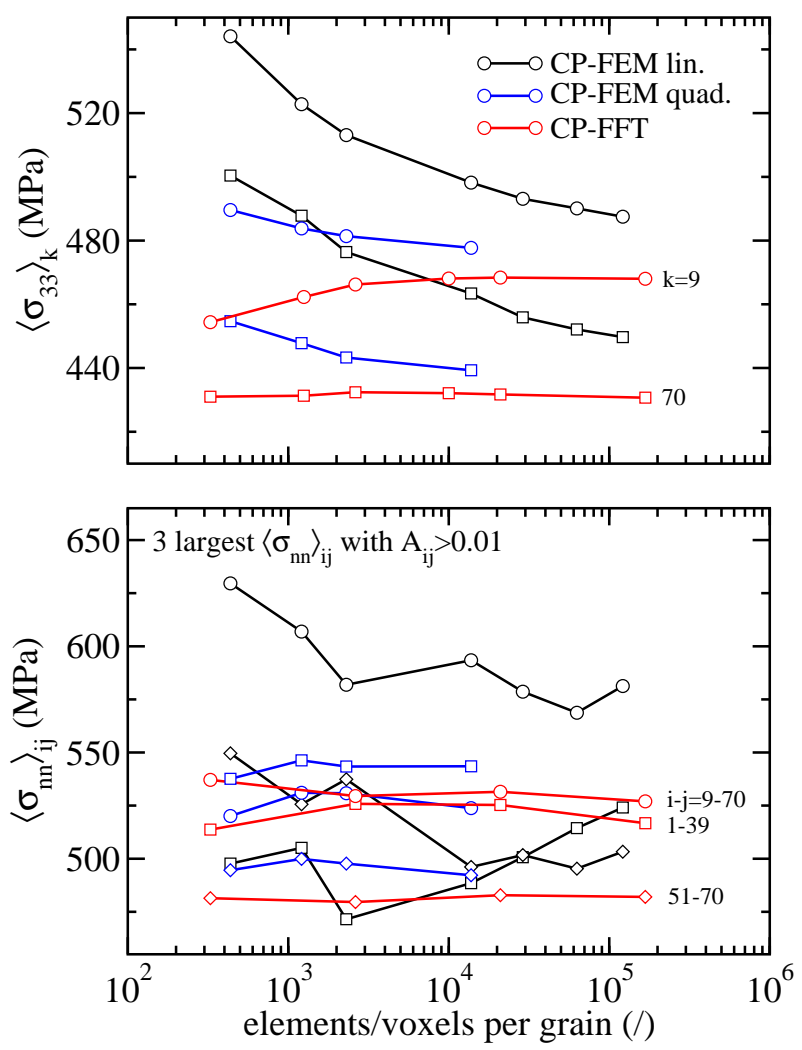

Figure 13: Top panel: mesh convergence analysis of grain-averaged axial stress $\left\langle\sigma_{33}\right\rangle_{k}$ calculated at 0.03 nominal strain in grains $k=9$ and 70 of the polycrystal model. A comparison is shown between linear mesh CP-FEM (in black), quadratic mesh CP-FEM (in blue) and CP-FFT (in red) simulations. Symbols denote grain labels $k$. Bottom panel: mesh convergence analysis of the three largest $\left\langle\sigma_{n n}\right\rangle_{i j}$ calculated on sufficiently large interfaces (with area $A_{i j}>0.01$ ) of the polycrystal model. Same color scheme is used as above. Symbols denote grain pair labels $i-j$ at the grain boundary interface. 


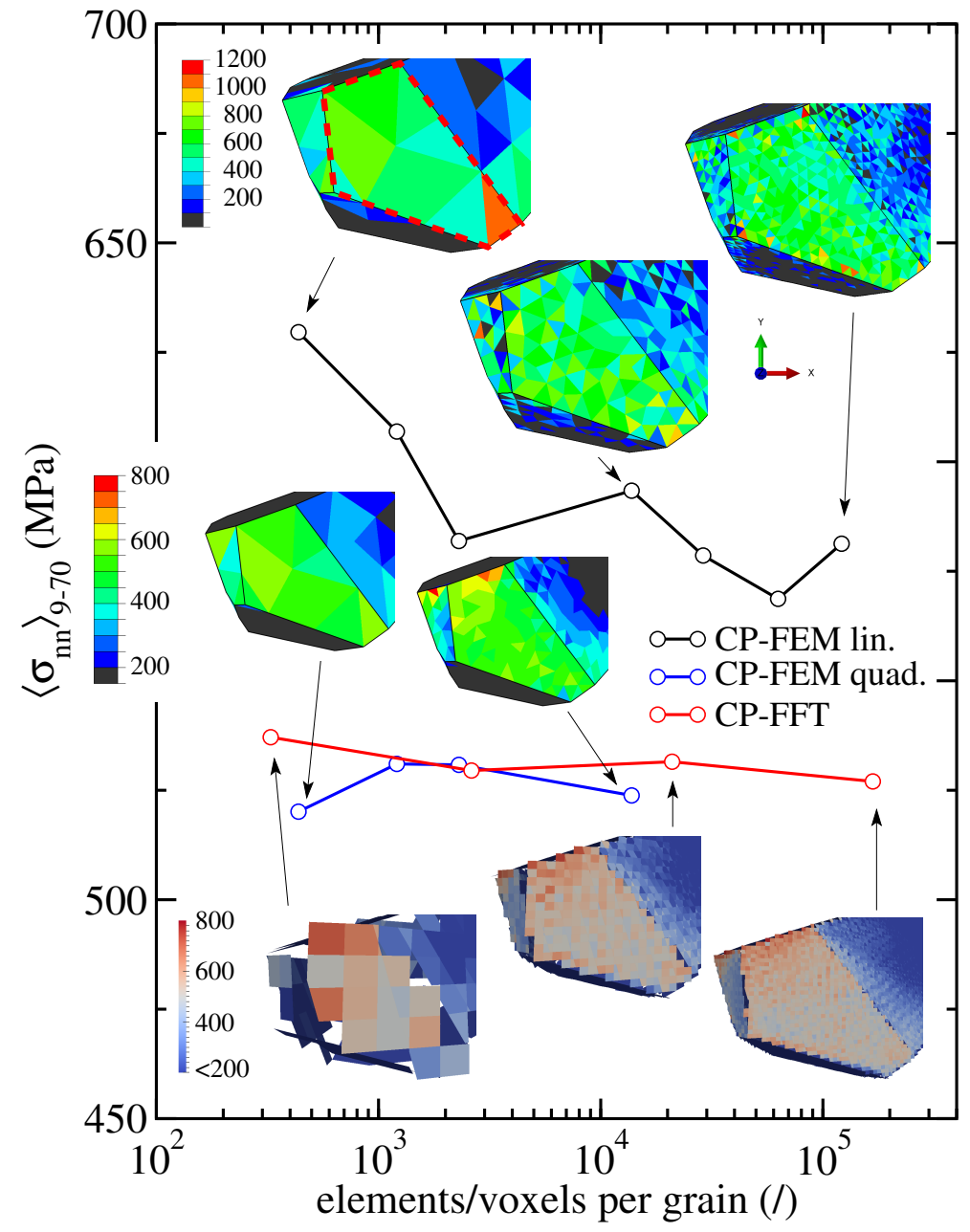

Figure 14: Mesh convergence analysis of average grain boundary normal stress $\left\langle\sigma_{n n}\right\rangle_{9-70}$ calculated at 0.03 nominal strain on the grain boundary between grains 9 and 70 for linear mesh CP-FEM, quadratic mesh CP-FEM and CP-FFT. The corresponding stress fields $\sigma_{n n}$ are shown in insets on grain 9. The 9-70 grain boundary is denoted by a red dashed line in the top-left inset. 


\begin{tabular}{rrrrrrl}
\hline \hline$i$ & $N_{g r}$ & $N_{e l}$ & $\dot{E}_{33}(1 / s)$ & $\Delta t_{m a x}(s)$ & NLGEOM & comment \\
\hline 1 & 1 & 497 & 0.0003 & 0.1 & 0 & $(\alpha, \beta, \gamma)=\left(10^{\circ}, 20^{\circ}, 30^{\circ}\right)$ \\
2 & 1 & 497 & 0.0003 & 0.1 & 0 & $(\alpha, \beta, \gamma)=\left(50^{\circ}, 100^{\circ}, 200^{\circ}\right)$ \\
\hline 3 & 2 & 3196 & 0.0003 & 0.1 & 0 & $\mathrm{C} 3 \mathrm{D} 4, \mathrm{C} 3 \mathrm{D} 10$ \\
4 & 2 & 25773 & 0.0003 & 0.1 & 0 & $\mathrm{C} 3 \mathrm{D} 4, \mathrm{C} 3 \mathrm{D} 10$ \\
5 & 2 & 165265 & 0.0003 & 0.1 & 0 & $\mathrm{C} 3 \mathrm{D} 4, \mathrm{C} 3 \mathrm{D} 10$ \\
6 & 2 & 268789 & 0.0003 & 0.1 & 0 & $\mathrm{C} 3 \mathrm{D} 4, \mathrm{C} 3 \mathrm{D} 10$ \\
7 & 2 & 1574978 & 0.0003 & 0.02 & 0 & $\mathrm{C} 3 \mathrm{D} 4$ \\
8 & 2 & 1574978 & 0.0003 & 0.05 & 0 & $\mathrm{C} 3 \mathrm{D} 4$ \\
9 & 2 & 1574978 & 0.0003 & 0.1 & 0 & $\mathrm{C} 3 \mathrm{D} 4, \mathrm{C} 3 \mathrm{D} 10$ \\
10 & 2 & 1574978 & 0.0003 & 0.1 & 1 & $\mathrm{C} 3 \mathrm{D} 4$ \\
11 & 2 & 1574978 & 0.0003 & 0.2 & 0 & $\mathrm{C} 3 \mathrm{D} 4$ \\
12 & 2 & 1574978 & 0.0003 & 0.5 & 0 & $\mathrm{C} 3 \mathrm{D} 4$ \\
13 & 2 & 1574978 & 0.0003 & 1 & 0 & $\mathrm{C} 3 \mathrm{D} 4$ \\
14 & 2 & 1574978 & 0.003 & 0.01 & 0 & $\mathrm{C} 3 \mathrm{D} 4$ \\
15 & 2 & 1574978 & 0.03 & 0.001 & 0 & $\mathrm{C} 3 \mathrm{D} 4$ \\
16 & 2 & 2490495 & 0.0003 & 0.1 & 0 & $\mathrm{C} 3 \mathrm{D} 4$ \\
17 & 2 & 5660585 & 0.0003 & 0.1 & 0 & $\mathrm{C} 3 \mathrm{D} 4$ \\
18 & 2 & 13140549 & 0.0003 & 0.1 & 0 & $\mathrm{C} 3 \mathrm{D} 4$ \\
\hline 19 & 100 & 43612 & 0.0003 & 0.1 & 0 & $\mathrm{C} 3 \mathrm{D} 4, \mathrm{C} 3 \mathrm{D} 10$ \\
20 & 100 & 120943 & 0.0003 & 0.1 & 0 & $\mathrm{C} 3 \mathrm{D} 4, \mathrm{C} 3 \mathrm{D} 10$ \\
21 & 100 & 229859 & 0.0003 & 0.1 & 0 & $\mathrm{C} 3 \mathrm{D} 4, \mathrm{C} 3 \mathrm{D} 10$ \\
22 & 100 & 1377694 & 0.0003 & 0.1 & 0 & $\mathrm{C} 3 \mathrm{D} 4, \mathrm{C} 3 \mathrm{D} 10$ \\
23 & 100 & 1377694 & 0.0003 & 0.1 & 1 & $\mathrm{C} 3 \mathrm{D} 4$ \\
24 & 100 & 1377694 & 0.003 & 0.01 & 0 & $\mathrm{C} 3 \mathrm{D} 4$ \\
25 & 100 & 1377694 & 0.03 & 0.001 & 0 & $\mathrm{C} 3 \mathrm{D} 4$ \\
26 & 100 & 2891536 & 0.0003 & 0.1 & 0 & $\mathrm{C} 3 \mathrm{D} 4$ \\
\hline \hline & 100 & 6283549 & 0.0003 & 0.1 & 0 & $\mathrm{C} 3 \mathrm{D} 4$ \\
\hline 100 & 12142329 & 0.0003 & 0.1 & 0 & $\mathrm{C} 3 \mathrm{D} 4$ \\
\hline
\end{tabular}

Table B.3: All the cases considered in the CP-FEM simulations. $N_{g r}$ denotes number of grains, $N_{e l}$ number of finite elements, $\dot{E}_{33}$ nominal strain rate, $\Delta t_{\max }$ maximum allowed simulation time step, NLGEOM Abaqus switch for non-linear geometry update and $\alpha, \beta, \gamma$ Euler angles. C3D4 and C3D10 denote linear and quadratic tetrahedral meshes, respectively. 


\begin{tabular}{rrrrrrl}
\hline \hline$i$ & $N_{g r}$ & $N_{v o}$ & $\dot{E}_{33}(1 / s)$ & $\Delta t(s)$ & TOL & comment \\
\hline 1 & 1 & 8 & 0.0003 & 0.1 & 0.001 & $(\alpha, \beta, \gamma)=\left(10^{\circ}, 20^{\circ}, 30^{\circ}\right)$ \\
2 & 1 & 8 & 0.0003 & 0.1 & 0.001 & $(\alpha, \beta, \gamma)=\left(50^{\circ}, 100^{\circ}, 200^{\circ}\right)$ \\
\hline 3 & 2 & 512 & 0.0003 & 0.1 & 0.001 & \\
4 & 2 & 4096 & 0.0003 & 0.1 & 0.001 & \\
5 & 2 & 32768 & 0.0003 & 0.1 & 0.001 & \\
6 & 2 & 125000 & 0.0003 & 0.1 & 0.001 & \\
7 & 2 & 262144 & 0.0003 & 0.1 & 0.001 & \\
8 & 2 & 1000000 & 0.0003 & 0.1 & 0.001 & \\
9 & 2 & 2097152 & 0.0003 & 0.01 & 0.001 & \\
10 & 2 & 2097152 & 0.0003 & 0.1 & 0.001 & \\
11 & 2 & 2097152 & 0.0003 & 0.1 & 0.0001 & \\
12 & 2 & 2097152 & 0.003 & 0.01 & 0.001 & \\
13 & 2 & 2097152 & 0.03 & 0.001 & 0.001 & \\
14 & 2 & 16777216 & 0.0003 & 0.1 & 0.001 & \\
\hline 15 & 100 & 4096 & 0.0003 & 0.1 & 0.001 & \\
16 & 100 & 32768 & 0.0003 & 0.1 & 0.001 & \\
17 & 100 & 125000 & 0.0003 & 0.1 & 0.001 & \\
18 & 100 & 262144 & 0.0003 & 0.1 & 0.001 & \\
19 & 100 & 1000000 & 0.0003 & 0.1 & 0.001 & \\
20 & 100 & 2097152 & 0.0003 & 0.01 & 0.001 & \\
21 & 100 & 2097152 & 0.0003 & 0.1 & 0.001 & \\
22 & 100 & 2097152 & 0.0003 & 0.1 & 0.0001 & \\
23 & 100 & 2097152 & 0.003 & 0.01 & 0.001 & \\
24 & 100 & 2097152 & 0.03 & 0.001 & 0.001 & \\
25 & 100 & 16777216 & 0.0003 & 0.1 & 0.001 & \\
\hline \hline & & & & & & \\
\hline
\end{tabular}

Table B.4: All the cases considered in the CP-FFT simulations. $N_{g r}$ denotes number of grains, $N_{v o}$ number of voxels, $\dot{E}_{33}$ nominal strain rate, $\Delta t$ simulation time step, TOL numerical tolerance and $\alpha, \beta, \gamma$ Euler angles. 

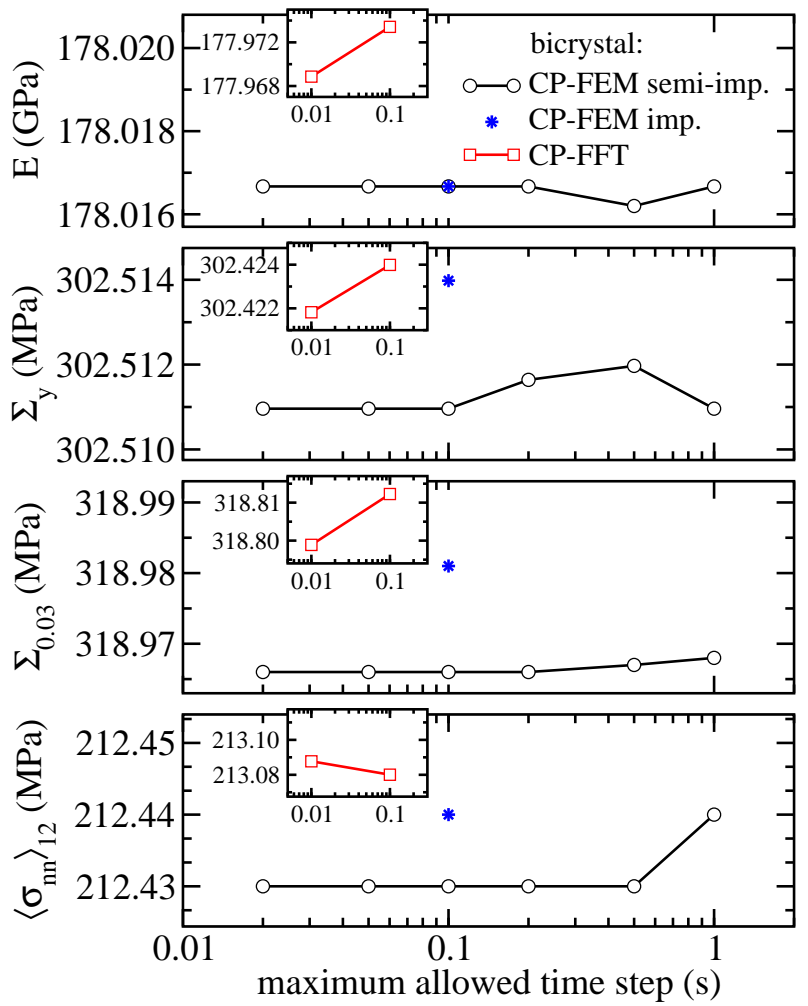

Figure C.15: Influence of simulation time step on various macroscopic quantities calculated in the bicrystal model. A comparison is shown between the CP-FEM (circles) and CP-FFT (squares, shown in insets) simulations. In the CP-FEM simulations, a time step $\Delta t$ was fixed indirectly by fixing the maximum allowed time step $\Delta t_{\max }$ so that $\Delta t<\Delta t_{\max }$ (however, for small $\left.\Delta t_{\max }, \Delta t \sim \Delta t_{\max }\right) .\left\langle\sigma_{n n}\right\rangle_{12}$ was calculated at 0.03 nominal strain. CP-FEM results of a fully-implicit time integration scheme (stars) are shown for comparison. Strain rate: $310^{-4} / \mathrm{s}$. 